\title{
EQUIVARIANT MORSE THEORY FOR STARSHAPED HAMILTONIAN SYSTEMS
}

\author{
CLAUDE VITERBO
}

\begin{abstract}
Let $\Sigma$ be a starshaped hypersurface in $R^{2 n}$; the problem of finding closed characteristics of $\Sigma$ can be classically reduced to a variational problem. This leads to studying an $S^{1}$-equivariant functional on a Hilbert space. The equivariant Morse theory of this functional, together with the assumption that $\Sigma$ only has finitely many geometrically distinct characteristics, leads to a remarkable formula relating the average indices of the characteristics. Using this formula one can prove, at least for $n$ even, that generically there are infinitely many characteristics (cf. [E1] for the convex case).
\end{abstract}

\section{NotATION}

$(x, y),|x|$

$\langle u, v\rangle,\|u\|$

$H_{*}(X), H^{*}(X)$

$H_{S^{1}, *}(X), H_{S^{1}}^{*}(X)$

$W^{1,2}\left(\mathbf{R} / T \mathbf{Z} ; \mathbf{R}^{2 n}\right)$

$\mathscr{C}^{k}$ scalar product and norm in $\mathbf{R}^{2 n}$ scalar product and norm in $L^{2}\left(\mathbf{R}^{2 n}\right)$ homology, cohomology of $X$ with rational coefficients $S^{1}$-equivariant homology, cohomology in the sense of Borel [Bo 2] with rational coefficients Sobolev space of continuous maps from $\mathrm{R} / T \mathrm{Z}$ into $\mathbf{R}^{2 n}$ with $L^{2}$ derivative

Banach space of $C^{k}$ maps

\section{INTRODUCTION, STATEMENT OF THE MAIN THEOREMS}

Let $\Sigma$ be a $C^{3}$ hypersurface of $\mathbf{R}^{2 n}$ strictly starshaped with respect to the origin.

We shall consider the closed characteristics of $\Sigma$, that is, the periodic solutions of

$$
\dot{x}=J N(x)
$$

where $N(x)$ is the outward normal, normalized by the condition $N(x) \cdot x=1$ (this will prove to be more convenient than the usual condition $|N(x)|=1$ ) and $J=\left(\begin{array}{cc}0 & I_{n} \\ -I_{n} & 0\end{array}\right)$ is the standard symplectic matrix.

Received by the editors October 7, 1987.

1980 Mathematics Subject Classification (1985 Revision). Primary 58F05; Secondary 34C25, $58 \mathrm{E} 99$.

Key words and phrases. Hamiltonian systems, closed characteristics, periodic orbits. 
The main known results are:

$(\mathscr{N})$ has always at least one periodic solution (cf. Rabinowitz in [R1]).

$(\mathscr{N})$ has at least $n$ geometrically distinct periodic solutions if $\Sigma$ satisfies some geometrical "pinching" condition (cf. [BLMR] generalizing a previous result of Ekeland and Lasry in [E-L] in the convex case). One of the results of this paper is

Theorem 1.1. In the $C^{k}$ topology (for $k \geq 3$ ), the following holds for a generic $\Sigma$ : Either $(\mathscr{N})$ has infinitely many primitive periodic solutions, or they are all hyperbolic. Moreover, the latter cannot occur for $n$ even.

By primitive solution, we mean one which is not the iterate of some other periodic solution.

The proof of 1.1 makes use of Theorem 1.2, which we shall state shortly.

Before stating it, we recall that in [V1, V2] we defined an index for a periodic solution of $(\mathscr{N})$. (This index, which generalized the one defined by Ekeland in [E1] for the convex case, is up to a constant the same as that defined by Amann, Conley, and Zehnder in [A-Z, C-Z]. For a proof of this statement see [Br] or [V3].)

If $i_{k}$ is the index of the $k$-iterate of a primitive solution, then the limit $\lim _{k \rightarrow+\infty} i_{k} / k$ exists and is denoted by $\hat{\imath}$ (cf. $\S 6$ ).

Also, we shall say that a primitive periodic orbit of $(\mathscr{N})$ is nondegenerate if and only if its nonzero Floquet multipliers are irrational, and zero is a simple multiplier (see $\S 6$ for the definitions). Then

Theorem 1.2. If $(\mathcal{N})$ has only finitely many nondegenerate primitive periodic orbits, then the following relations hold:

$$
\begin{aligned}
& \sum^{\prime} \frac{(-1)^{i_{1}}}{i}+\frac{1}{2} \sum^{\prime \prime} \frac{(-1)^{i_{1}}}{i}=\frac{1}{2}, \\
& \sum^{\prime \prime \prime} \frac{(-1)^{i_{1}}}{\hat{\imath}}+\frac{1}{2} \sum^{(\mathrm{iv})} \frac{(-1)^{i_{1}}}{\hat{\imath}}=0,
\end{aligned}
$$

where $\Sigma^{\prime}$ (resp. $\left.\Sigma^{\prime \prime}\right)$ is the sum extended over all primitive orbits with positive (resp. negative) $\hat{\imath}$ such that $j(-1)=i_{2}-i_{1}$ is even, and $\sum^{\prime \prime}\left(\right.$ resp. $\left.\sum^{(\mathrm{iv})}\right)$ those with positive (resp. negative) $\hat{i}$ such that $j(-1)=i_{2}-i_{1}$ is odd.

The aim of this paper is to prove Theorem 1.2. Let us prove Theorem 1.1 assuming Theorem 1.2.

According to a result of Takens (cf. [Ta]) for generic $\Sigma$, all the primitive periodic orbits are nondegenerate. Moreover, his method can be extended, using multijet spaces instead of jet spaces, to show that generically, no resonance relation, in the terminology of [E1], between finitely many orbits occurs. In $\S 6$ we shall define resonance relations, and we shall see that if there exists a nonhyperbolic orbit, then (1.3) or (1.4) is a resonance relation. The fact that for $n$ even there must be a nonhyperbolic orbit is proved in $\S 9$. 
Let us now explain the relation between this paper and [E1]. In [E1] Theorem 1.1 is proved for $\Sigma$ convex. Moreover, there cannot be finitely many hyperbolic orbits (whether $n$ is even or odd). The proof uses Morse theory as extended by Bott to functions with nondegenerate critical manifolds.

On the other hand, in order to prove (1.3) or (1.4) we need equivariant Morse theory. Even in the convex case [E1] asserts that if there are only finitely many orbits, they satisfy a resonance relation. Our result improves this by specifying that this relation is actually (1.3). (As we shall see in the convex case, $\hat{\imath}$ is always positive, so (1.4) is obviously satisfied.)

This paper is organized as follows:

In $\S 2$, we reduce our problem to a fixed period Hamiltonian system.

In $\S 3$ we put this in a variational form by using the dual action functional (cf. [C] and [C-E]) as generalized in [E2] and [BLMR]. We also define a finitedimensional reduction in order to avoid some technical inconvenience. This reduction is $S^{1}$-equivariant and more like the one defined by Conley and Zehnder [C-Z] than the broken geodesic type of [E1]. In $\S 4$ we prove that this reduction satisfies condition (C) of Palais and Smale.

In $\S 5$ we compute the equivariant Poincaré series of $\left(X, X^{-c}\right)$ for $c$ large enough, and in $\S 6$ the contribution of the orbits of $(\mathscr{N})$ to the Morse series of our function $F$.

At this point one critical point of our function $F$. does not yield a solution of $(\mathscr{N})$, that is, the origin, and it is a very degenerate critical point. The aim of $\S 7$ is to show that we can "forget" the contribution of the origin to the Morse series of $F$.

Finally, in $\S 8$ we prove Theorem 1.2, and in $\S 9$ we show that for $n$ even, there cannot be finitely many hyperbolic orbits.

This paper is an extended version of my "thèse de $3^{\text {eme }}$ cycle." I would like to thank my advisor, Ivar Ekeland, for introducing me to Hamiltonian systems and for his help and encouragement. The reader will easily see how much this work owes to his. I would like to mention how enjoyable it is to work with him.

\section{ReDUCtion to A FIXED PERIOd HAMILTONIAN SYSTEM}

We recall that if $H$ is a function such that $\Sigma=H^{-1}(1)$ and 1 is a regular value for $H$, then the periodic solutions of $(\mathscr{N})$ coincide with those of $\dot{x}=$ $J \nabla H(x)$ on $\Sigma$.

Now let $\phi: \mathbf{R}_{+} \rightarrow \mathbf{R}$ be some function having 0 as its only critical point, and $j: \mathbf{R}^{2 n} \rightarrow \mathbf{R}$ be the gauge function of $\Sigma$, that is,

$$
j(\lambda x)=\lambda \text { for all } x \in \Sigma, \lambda \in \mathbf{R}_{+} .
$$

(This is well defined and $C^{3}$ in $\mathbf{R}^{2 n}-\{0\}$ since $\Sigma$ is strictly starshaped with respect to the origin.) Set $\widetilde{H}(x)=\phi(j(x))$, and consider the fixed period system

$$
(\widetilde{\mathscr{H}})\left\{\begin{array}{l}
\dot{x}=J \nabla \widetilde{H}(x), \\
x(0)=x(T)
\end{array}\right.
$$


Then we claim

Lemma 2.1. Nonzero solutions of $(\widetilde{\mathscr{H}})$ can be written as $x(t)=\rho y(t \tau / T)$ where $y$ is a $\tau$-periodic solution of $(\mathscr{N})$, and $\phi^{\prime}(\rho) / \rho=\tau / T$.

Proof. Straightfoward, as soon as one notices that $\widetilde{H}^{\prime}(x)=\phi^{\prime}(j(x)) j^{\prime}(x)$, and that on $\Sigma, j^{\prime}(x)=N(x)$.

We now need to specify $\phi$.

Lemma 2.2. For all real numbers a, large enough, there exists a function $\phi$ : $\mathbf{R}_{+} \rightarrow \mathbf{R}$ such that

$$
\begin{gathered}
\phi \text { is } C^{\infty}, \text { nonnegative, } \\
\phi(t)=\frac{a}{2} t^{2} \text { near the origin, }
\end{gathered}
$$

(2.5) $t \rightarrow \phi^{\prime}(t) / t$ is decreasing and goes to zero as $t$ goes to infinity.

Moreover, for such a choice of $\phi,(\widetilde{\mathscr{H}})$ has one nonzero solution for every periodic orbit of $(\mathcal{N})$ of period less than aT/2.

Proof. The first part is up to a few details Lemma II.2 of [E1]; the second part follows from our Lemma 2.1.

Finally, for technical reasons we want to deal with a Hamiltonian $H$ which coincides with $\frac{1}{2} \varepsilon|x|^{2}$ for $|x|$ large and which has the same $T$-periodic orbits as $\tilde{H}$.

Now, according to a theorem of Yorke (cf. [Y]), if $\left|H^{\prime \prime}(x)\right|<\varepsilon$ then ( $\mathscr{H}$ ) has no nontrivial solutions for $T$ such that $\varepsilon T<2 \pi$.

Let us compute $\widetilde{H}^{\prime \prime}(x)$ :

$$
\left(\tilde{H}^{\prime \prime}(x) y, y\right)=\phi^{\prime \prime}(j(x))\left(j^{\prime}(x) y\right)^{2}+\phi^{\prime}(j(x)) j^{\prime \prime}(x) .
$$

Then $\widetilde{H}^{\prime \prime}(x)$ goes to zero when $x$ goes to infinity since

$j^{\prime \prime}$ is homogeneous of degree minus one, and $\phi^{\prime}(t) / t$ goes to zero as $t$ goes to infinity (cf. 2.5). Thus $\phi^{\prime}(j(x)) j^{\prime \prime}(x)$ goes to zero as $x$ goes to infinity.

$j^{\prime}$ is homogeneous of degree zero, thus bounded, and $\phi^{\prime \prime}(t)$ goes to zero as $t$ goes to infinity (because $\phi^{\prime}(t) / t$ is decreasing so that $\left(\phi^{\prime}(t) / t\right)^{\prime}<0$, which implies $\left.\phi^{\prime \prime}(t)<\phi^{\prime}(t) / t\right)$, hence $\phi^{\prime \prime}(j(x)) j^{\prime}(x)^{2}$ goes to zero as $t$ goes to infinity.

Clearly enough, we can construct a function $H$, coinciding with $\widetilde{H}$ on $\{x \mid \tilde{H}(x) \leq A\}=U_{A}$ for some large $A$, with $\frac{1}{2} \varepsilon|x|^{2}$ outside some large ball, such that $\nabla H(x)$ does not vanish and $\left|H^{\prime \prime}(x)\right|<\varepsilon$ outside $U_{A}$. To construct $H$, one just has to take a convex combination of $\widetilde{H}$ and $\frac{1}{2} \varepsilon|x|^{2}$, that is, $H(x)=\lambda(x) \widetilde{H}(x)+(1-\lambda(x)) \frac{1}{2} \varepsilon|x|^{2}$, with $\lambda=1$ in a neighborhood of $U_{A}$ and $\lambda=0$ in a neighborhood of infinity. It is easy to check that $\left|H^{\prime \prime}(x)\right| \leq \varepsilon$ 
outside $U_{A}$, and $\nabla H(x) \neq 0$ follows from the fact that $\Sigma$ is starshaped, so that $\nabla \tilde{H}(x)$ and $\frac{1}{2} \varepsilon x$ both point outward.

Consider now a solution of $(\mathscr{H})$. Since its trajectory is in some energy level, it is either contained in $U_{A}$, in which case it is a solution of $(\widetilde{\mathscr{H}})$, or outside $U_{A}$. The latter cannot happen, because the condition $\left|H^{\prime \prime}(x)\right|<\varepsilon$ implies there is no nontrivial solution (by Yorke's theorem), and since $\nabla H(x) \neq 0$ outside $U_{A}$, there are no trivial solutions either. We thus proved

Proposition 2.7. For every large real number a, small $\varepsilon$, there exists a function $H$ on $\mathbf{R}^{2 n}$ such that

$$
\begin{gathered}
H \text { is } C^{1} \text { on } \mathbf{R}^{2 n} \text {, and } C^{3} \text { on } \mathbf{R}^{2 n}-\{0\}, \\
H=\widetilde{H} \text { in } U_{A} \text {, and } H(x)=\frac{1}{2} \varepsilon|x|^{2} \text { for }|x| \text { large },
\end{gathered}
$$

$(\mathscr{H})$ and $(\widetilde{\mathscr{H}})$ have the same set of solutions consisting of:

the constant solution at zero,

one solution for every periodic orbit of $(\mathcal{N})$ of period less than $a T / 2$

\section{The DUAL ACTION FUNCTIONAL AND ITS FINITE-DIMENSIONAL REDUCTION}

Let $H$ be a function on $\mathbf{R}^{2 n}$ such that $\nabla H$ is Lipschitz. Then for some large constant $K, H_{K}(x)=H(x)+\frac{1}{2} K|x|^{2}$ is a strictly convex function, that is,

$$
\left(\nabla H_{K}(x)-\nabla H_{K}(y), x-y\right) \geq \frac{\varepsilon}{2}|x-y|^{2}
$$

for all $x, y \in \mathbf{R}^{2 n}$, and some positive $\varepsilon$.

Let $H_{K}^{*}$ be the Fenchel dual of $H_{K}$, that is,

$$
H_{K}^{*}(y)=\sup _{x \in \mathbf{R}^{2 n}}\left[(x, y)-H_{K}(x)\right],
$$

and define

$$
F_{K}(x)=\int_{0}^{T}\left[\frac{1}{2}(J \dot{x}-K x, x)+H_{K}^{*}(-J \dot{x}+K x)\right] d s
$$

for $x \in X=W^{1,2}\left(\mathbf{R} / T \mathbf{Z} ; \mathbf{R}^{2 n}\right)$.

It is well known that

Proposition 3.4. Assume $K T \notin 2 \pi \mathrm{Z}$; then $x$ is a critical point of $F_{K}$ if and only if it is a solution of $(\mathscr{H})$.

Proof. Straightforward computation (see [BLMR]).

The condition $K T \notin 2 \pi \mathrm{Z}$ is due to the fact that in this case the map $x \rightarrow-J \dot{x}+K x$ is a Hilbert space isomorphism between $X=W^{1,2}\left(\mathbf{R} / T \mathbf{Z}, \mathbf{R}^{2 n}\right)$ 
and $E=L^{2}\left(\mathbf{R} / T \mathbf{Z} ; \mathbf{R}^{2 n}\right)$. We shall denote its inverse by $M_{K}$, and the change of variable $x=M_{K} u$ yields the functional

$$
\Psi_{K}(u)=\int_{0}^{T}\left[-\frac{1}{2}\left(M_{K} u, u\right)+H_{K}^{*}(u)\right] d s
$$

for $u \in E$, which is sometimes easier to handle.

Throughout this chapter, we shall fix $K$, and write $F$ for $F_{K}, \Psi$ for $\Psi_{K}$ (but never $H$ for $H_{K}$ !).

Let us mention that, identifying $S^{1}$ and $\mathbf{R} / T \mathbf{Z}$, we consider the $S^{1}$ action on $X$ or $E$ defined by

$$
\theta \cdot u(\cdot)=u(\theta+\cdot)
$$

then $F$ and $\Psi$ are $S^{1}$-equivariant.

We shall now define a finite-dimensional reduction of $\Psi$. The main point is to remark that $F$ is convex in the direction orthogonal to some finitedimensional subspace that we shall now define.

Consider the quantity:

$$
\left\langle\Psi^{\prime}(u)-\Psi^{\prime}(v), u-v\right\rangle=\left\langle M(u-v)+\nabla H_{K}^{*}(u)-\nabla H_{K}^{*}(v), u-v\right\rangle .
$$

Since $\nabla H_{K}^{*}$ is strictly convex, we have

$$
\left\langle\nabla H_{K}^{*}(u)-\nabla H_{K}^{*}(v), u-v\right\rangle \geq \varepsilon\|u-v\|^{2} .
$$

Now if we consider $M$ as a map from $L^{2}$ to $L^{2}$ it is a selfadjoint compact operator (because the inclusion map of $W^{1,2}$ into $L^{2}$ is compact), so if $G$ is the subspace of $E$ generated by the eigenvectors of $M$ whose eigenvalue is less than $-\varepsilon / 2$, we have that for $u-v \in G^{\perp}$

$$
\left\langle M(u-v)+\nabla H_{K}^{*}(u)-\nabla H_{K}^{*}(v), u-v\right\rangle \geq \frac{\varepsilon}{2}\|u-v\|^{2} .
$$

Inequality (3.8) means that $\Psi$ is strictly convex in the direction of $G^{\perp}$. That is, for any $e \in E$ the function $h \rightarrow \Psi(e+h)$ defined on $G^{\perp}$ is strictly convex. We now prove

Proposition 3.9. The function $h \rightarrow \Psi(g+h)$ has a unique minimum in $G^{\perp}: h(g)$. If for $g \in G$ we set $\psi(g)=\Psi(g+h(g))$, then

$\psi$ is $C^{1}$ on $G$ and $S^{1}$ equivariant.

$g$ is a critical point on $\psi$ if and only if $g+h(g)$ is a critical (3.11) point of $\Psi$. Moreover, their critical values, index, and nullities (as defined in the proof) coincide.

If $g_{0} \in G$ and $H$ is $C^{k}(k \geq 2)$ in a neighborhood of the (3.12) trajectory of $g_{0}+h\left(g_{0}\right)$, then $\psi$ is $C^{k-1}$ in a neighborhood of $g_{0}$.

Proof. To begin with let us show that $g \rightarrow h(g)$ is Lipschitz. 
We first remark that $\Psi$ is $C^{1}$, and $h(g)$ is defined by

$$
\frac{\partial}{\partial h} \Psi(g+h(g))=0 \text {. }
$$

Now let $g_{1}, g_{2} \in E$ and set $h_{1}=h\left(g_{1}\right), h_{2}=h\left(g_{2}\right)$. Then by (3.13), since $h_{1}-h_{2} \in G^{\perp}$,

$$
\left\langle\Psi^{\prime}\left(g_{1}+h_{1}\right)-\Psi^{\prime}\left(g_{2}+h_{2}\right), h_{1}-h_{2}\right\rangle=0
$$

On the other hand

$$
\left\langle\Psi^{\prime}\left(g_{1}+h_{2}\right)-\Psi^{\prime}\left(g_{1}+h_{1}\right), h_{2}-h_{1}\right\rangle \geq \frac{1}{2} \varepsilon\left\|h_{2}-h_{1}\right\|^{2}
$$

by convexity of $\Psi$ in the direction of $G^{\perp}$, and

$$
\left\|\Psi^{\prime}\left(g_{1}+h_{2}\right)-\Psi^{\prime}\left(g_{2}+h_{2}\right)\right\| \leq C\left\|g_{1}-g_{2}\right\|
$$

because $M$ is linear and $\nabla H_{K}^{*}$ is Lipschitz.

To prove this last statement, we use the equality $\nabla H_{K}^{*}\left(\nabla H_{K}(x)\right)=x$. Thus $\nabla H_{K}^{*}\left(y_{1}\right)-\nabla H_{K}^{*}\left(y_{2}\right)=x_{1}-x_{2}$ where $\nabla H_{K}\left(x_{1}\right)=y_{1}, \nabla H_{K}\left(x_{2}\right)=y_{2}$. By strict convexity of $\nabla H_{K},\left|\nabla H_{K}\left(x_{1}\right)-\nabla H_{K}\left(x_{2}\right)\right| \geq \frac{1}{2} \varepsilon\left|x_{1}-x_{2}\right|$; that is, $\left|x_{1}-x_{2}\right| \leq$ $(2 C / \varepsilon)\left|y_{1}-y_{2}\right|$, so $\nabla H_{K}^{*}$ is Lipschitz.

Combining (3.14), (3.15), and (3.16) we get

$$
\frac{1}{2} \varepsilon\left\|h_{2}-h_{1}\right\|^{2} \leq C\left\|g_{1}-g_{2}\right\|\left\|h_{1}-h_{2}\right\| ;
$$

that is, $\left\|h_{2}-h_{1}\right\| \leq(2 C / \varepsilon)\left\|g_{1}-g_{2}\right\|$ and $h$ is Lipschitz.

Using this, we prove that $\psi$ is $C^{1}$ and that

$$
d \psi(g)=d \Psi(g+h(g))=\frac{\partial}{\partial g} \Psi(g+h(g)) .
$$

We compute

$$
\begin{aligned}
\psi(g+\delta g)-\psi(g)= & \Psi(g+\delta g+h(g+\delta g))-\Psi(g+h(g)) \\
= & \frac{\partial}{\partial g} \Psi(g+h(g)) \delta g+\frac{\partial}{\partial h} \Psi(g+h(g))(h(g+\delta g)-h(g)) \\
& +O(\|\delta g\|+\|h(g+\delta g)-h(g)\|) .
\end{aligned}
$$

The map $g \rightarrow h(g)$ being Lipschitz,

$$
O(\|h(g+\delta g)-h(g)\|)=O(\|\delta g\|)
$$

and since $\partial \Psi(g+h(g)) / \partial h=0$, this leads to (3.17).

As for $S^{1}$ equivariance, since $G$ is invariant by $S^{1}$ and $\Psi$ is equivariant, $g \rightarrow h(g)$ is equivariant, hence also $\psi$.

The first part of (3.11) follows immediately from (3.17) and also the equality of critical levels.

We shall now define what is meant by the index of a solution. If $g \in G$ and $\psi$ is $C^{2}$ in a neighborhood of $g$, the index of $g$ is the Morse index of $\psi$, that is, the index of the quadratic form $\psi^{\prime \prime}(g)$. A similar definition does not work 
for $\Psi$ since $\Psi$ is never $C^{2}$; our index will then be the index of the quadratic form, still denoted by $\Psi^{\prime \prime}(g+h(g))$ :

$$
(Q v, v)=\int_{0}^{T}\left[(M v, v)+\left(H_{K}^{* \prime \prime}(g+h(g)) v, v\right)\right] d s .
$$

Similar definitions hold for nullity.

Now $\psi^{\prime \prime}(g)$ can be identified with the restriction of $Q$ to the tangent space $T$ of $\{(g, h(g)) \mid g \in G\}$. But it follows by taking the derivative of (3.13) that this tangent space is orthogonal for $Q$ to $G^{\perp}$. By convexity of $\Psi$ in the direction $G^{\perp}, Q$ is positive definite on $G^{\perp}$, so its index and nullity coincide with the index and nullity of its restriction to $T$.

We finally prove (3.12).

Equation (3.13) can be written more explicitly as

$$
M h-P \nabla H_{K}^{*}(g+h)=0
$$

where $P$ is the orthogonal projection on $G^{\perp}$.

From (3.18) we see, using the fact that $\nabla H_{K}^{*}$ is Lipschitz, that $P \nabla H_{K}^{*}(g+h)$ is in $L^{2}$, so $M h$ is in $L^{2}$, and $H$ is in $W^{1,2} \subset \mathscr{C}^{0}$. By induction, we see that if $H$ is $C^{k}, \nabla H_{K}^{*}$ is $C^{k-1}$, and so is $M h$, whence $h$ is in $\mathscr{C}^{k}\left(\mathbf{R} / T Z ; \mathbf{R}^{2 n}\right)$.

We now wish to show, assuming $H$ is $C^{k}$ in a neighborhood of the trajectory of $g_{0}+h\left(g_{0}\right)$, that $g \rightarrow h(g)$ is $C^{k}$ in a neighborhood of $g_{0}$, as a map from $G$ into $\mathscr{C}^{0}\left(\mathbf{R} / T Z ; \mathbf{R}^{2 n}\right)$. Since $\Psi$ is $C^{k}$ in a neighborhood of $g_{0}+h\left(g_{0}\right)$ in $\mathscr{C}^{0}\left(\mathbf{R} / T Z ; \mathbf{R}^{2 n}\right)$, this will imply (3.12).

Our first step will be to show that $g \rightarrow h(g)$ (through the end of this proof, this map will be considered as a map from $G$ to $\left.\mathscr{C}^{0}\left(\mathbf{R} / T Z ; \mathbf{R}^{2 n}\right)\right)$ is continuous.

Let $g_{n} \in G$ be a sequence with limit $g$, and $h_{n}=h\left(g_{n}\right)$. Then $h_{n} \rightarrow h$ in $L^{2}$ (since $g \rightarrow h(g)$ is Lipschitz when considered as a map into $L^{2}$ ) so $M h_{n} \rightarrow M h$ in $W^{1,2}$.

But by (3.18) $M h_{n}=P \nabla H_{K}^{*}\left(g_{n}+h_{n}\right)$, so $P \nabla H_{K}^{*}\left(g_{n}+h_{n}\right) \rightarrow M h$ in $W^{1,2}$; that is, we can write

$$
\nabla H_{K}^{*}\left(g_{n}+h_{n}\right)=M h_{n}+r_{n} \text { with } r_{n} \in G .
$$

By continuity of $g \rightarrow h(g)$ in $L^{2}$ and since $\nabla H_{K}^{*}$ is Lipschitz, the left-hand side of (3.19) has a limit in $L^{2}$; thus $r_{n}$ has a limit $r \in G$ (in any topology, since $G$ is finite dimensional). Therefore $M h_{n}+r_{n}$ has a limit in $\mathscr{C}^{0}$, and since $z \rightarrow \nabla H_{K}(z)$ defines a continuous map on $C^{0}, g_{n}+h_{n}=\nabla H_{K}\left(M h_{n}+r_{n}\right)$ has a limit in $\mathscr{C}^{0}$, and this limit must be $g+h$, since it has to coincide with the $L^{2}$ limit. This proves continuity.

We can now assert that the image of a neighborhood of $g_{0}$ by $h$ will be contained in a $C^{0}$ neighborhood of $h\left(g_{0}\right)$, so the trajectories of $g+h(g)$ are in a neighborhood of $g_{0}+h\left(g_{0}\right)$, where $H$ is assumed to be $C^{k}$. 
In order to prove that $g \rightarrow h(g)$ is $C^{k}$ (as a map into $\mathscr{C}^{0}$ ) in a neighborhood of $g_{0}$, let us consider the map $\phi: G \times\left(G^{\perp} \cap \mathscr{C}^{0}\right) \rightarrow G^{\perp} \cap \mathscr{C}^{0}$ defined by $\phi(g, h)=M h-P \nabla H_{K}^{*}(g+h)$. Then $h(g)$ is defined by equation (4.18). That is, $\phi(g, h(g))=0$. By the implicit function theorem, if $(\partial \phi / \partial h)\left(g_{0}, h\left(g_{0}\right)\right)$ is invertible and $\phi$ is $C^{k-1}$, then $g \rightarrow h(g)$ is $C^{k}$ in a neighborhood of $g_{0}$. This would prove (4.12). We see that $(\partial \phi / \partial h)(g, h)=M-P H_{K}^{* \prime \prime}(g+h)$. If we consider this as a map between $L^{2}$ spaces, it will be invertible, since for $v \in G^{\perp}$

$$
\begin{aligned}
& \int_{0}^{T}\left(M v-P H_{K}^{*}(g+h) v, v\right) d s \\
& \quad=\int_{0}^{T}\left[(M v, v)-\left(H_{K}^{*}(g+h) v, v\right)\right] d s<-\frac{\varepsilon}{2}\|v\|^{2},
\end{aligned}
$$

i.e., $M-P H_{K}^{* \prime \prime}(g+h)$ is a selfadjoint negative definite operator. Now if $M v-$ $P H_{K}^{* \prime \prime}(g+h) v$ is in $\mathscr{C}^{0}$, then (since $\left.M v \in W^{1,2} \subset \mathscr{C}^{0}\right) \quad P H_{K}^{* \prime \prime}(g+h) v \in \mathscr{C}^{0}$, hence $H_{K}^{* \prime \prime}(g+h) v \in \mathscr{C}^{0}$, and finally, since $H_{K}^{\prime \prime}\left(H_{K}^{*}(g+h)\right)=\left[H_{K}^{* \prime \prime}(g+h)\right]^{-1}$ is continuous, $v \in \mathscr{C}^{0}$. Thus the inverse image of $\mathscr{C}^{0}$ by $M-P H_{K}^{* \prime \prime}(g+h)$ is in $\mathscr{C}^{0}$, and this map is an isomorphism on $L^{2}$, so it is one on $\mathscr{C}^{0}$.

Finally, it is easy to check that if $H$ is $C^{k}, H_{K}^{*}$ is $C^{k}$, and $\phi$ is $C^{k-1}$, thus proving (3.12).

\section{The Palais-Smale condition is Satisfied By $\psi$}

As the reader might have guessed from the heading of the section, we wish to prove

Proposition 4.1. $\psi$ satisfies condition (C) of Palais and Smale; furthermore, if $g_{n} \in G$ is a sequence such that $\psi^{\prime}\left(g_{n}\right) \rightarrow 0$, then $g_{n}$ has a converging subsequence.

Proof. To begin with, since $G$ is finite dimensional, we only need to show that $g_{n}$ is bounded. We argue by contradiction and assume $\left|g_{n}\right|$ goes to infinity. Then $M g_{n}$ goes to infinity, and $z_{n}=M g_{n}+M h\left(g_{n}\right)$ goes to infinity in the $\mathscr{C}^{0}$ topology (since $M: L^{2} \rightarrow W^{1,2} \subset \mathscr{C}^{0}$ is continuous and the projection on $G$ is continuous on $\left.\mathscr{C}^{0}\right)$.

Now $\psi^{\prime}\left(g_{n}\right) \rightarrow 0$ can be written

$$
M\left(g_{n}+h_{n}\right)-\nabla H_{K}^{*}\left(g_{n}+h_{n}\right)=\varepsilon_{n},
$$

where $\varepsilon_{n} \in G$ goes to zero. This implies

$$
\nabla H_{K}\left(z_{n}-\varepsilon_{n}\right)+J \dot{z}_{n}-K z_{n}=0,
$$

that is,

$$
J \dot{z}_{n}+\nabla H\left(z_{n}-\varepsilon_{n}\right)=K \varepsilon_{n} .
$$


Since $z_{n}$ goes to infinity in $\mathscr{C}^{0}$, there is a sequence $t_{n} \in[0, T]$ such that $\left|z_{n}\left(t_{n}\right)\right|$ goes to infinity. Changing $z_{n}$ into $z_{n}\left(t_{n}+\cdot\right)$, we can assume that $\left|z_{n}(0)\right|$ goes to infinity.

Now let $n$ be large enough so that $\left|\varepsilon_{n}\right|_{C^{0}}<R / 2 K T,\left|z_{n}(0)\right|>3 R$, where $H(x)=\frac{1}{2} \varepsilon|x|^{2}$ outside a ball of radius $R$, as in (2.9) of Proposition 2.7; we wish to show that $\left|z_{n}(t)\right| \geq R$ for all $t$ 's.

We argue by contradiction; let $\tau_{n}$ be the first time that $z_{n}$ enters $B(0, R)$, thus $\left|z_{n}(t)\right| \geq R$ on $\left[0, \tau_{n}\right]$. As long as $t$ is in $\left[0, \tau_{n}\right],(4.2)$ yields

$$
J \dot{z}_{n}+\varepsilon z_{n}=(K+\varepsilon) \varepsilon_{n}
$$

so that

$$
z_{n}(t)=(K+\varepsilon) \int_{0}^{t} \exp (\varepsilon J s) \varepsilon_{n} d s+\exp (\varepsilon J S) z_{n}(0)
$$

Thus

but

$$
z_{n}\left(\tau_{n}\right)=(K+\varepsilon) \int_{0}^{\tau_{n}} \exp (\varepsilon J s) \varepsilon_{n}(s) d s+\exp \left(\varepsilon J \tau_{n}\right) z_{n}(0)
$$

$$
(K+\varepsilon)\left|\int_{0}^{\tau_{n}} \exp (\varepsilon J s) \varepsilon_{n}(s) d s\right| \leq(K+\varepsilon) \tau_{n} R / 2 K T \leq R
$$

so $\left|z_{n}\left(\tau_{n}\right)\right| \geq\left|z_{n}(0)\right|-R \geq 2 R$, a contradiction since we assumed $\left|z_{n}\left(\tau_{n}\right)\right|=R$.

Now since $\left|z_{n}(t)\right| \geq R$ for all $t$ 's, (4.3) and thus (4.4) hold for all $t$ 's. From (4.4) we infer

$$
z_{n}(T)-z_{n}(0)=(K+\varepsilon) \int_{0}^{T} \exp (\varepsilon J s) \varepsilon_{n}(s) d s+(\exp (\varepsilon J T)-I) z_{n}(0),
$$

hence

$$
\left|z_{n}(T)-z_{n}(0)\right| \geq\left|(\exp (\varepsilon J T)-I) z_{n}(0)\right|-R,
$$

but if $0<\varepsilon T<2 \pi$, $(\exp \varepsilon J T-I)$ is a linear isomorphism, so $\mid(\exp (\varepsilon J T)-$ I) $z_{n}(0)|\geq C| z_{n}(0) \mid$, and finally

$$
\left|z_{n}(T)-z_{n}(0)\right| \geq C\left|z_{n}(0)\right|-R .
$$

For $\left|z_{n}(0)\right|$ large enough, the right-hand side is positive, yielding $\mid z_{n}(T)-$ $z_{n}(0) \mid>0$, a contradiction since $z_{n}$ is $T$-periodic.

\section{Computing $H_{S^{1}}^{*}\left(X, X^{-\infty}\right)$}

Up to now, we have been working mainly with $\Psi$ and $\psi$. In this section we need to work with $F$ and $f$. We have $F(M u)=\Psi(u)$, and since $G$ is preserved by $M, f(M u)=\psi(u)$. We call $Z$ the space $G$ when we consider it as the domain of $f$, and set for a real number $c$

$$
X^{c}=\{x \in X \mid F(x) \leq c\},
$$

$Z^{c}, E^{c}, G^{c}$ having the obvious meaning. It is clear that $M$ is a homeomorphism between $G^{c}$ and $Z^{c}$, as well as between $E^{c}$ and $X^{c}$. Moreover, we have the following lemma. 
Lemma 5.1. The natural embedding $Z^{c} \hookrightarrow X^{c}$ is an $S^{1}$-equivariant homotopy equivalence.

Proof. We actually shall prove this for the natural embedding $G^{c} \hookrightarrow E^{c}$. We recall that the natural embedding is given by $g \rightarrow g+h(g)$. Now if $e=g+h \in$ $E^{\mathcal{c}}$ set $r_{t}(e)=g+(1-t) h+t h(g)$. Then by convexity in the direction of $G^{\perp}$, we have

$$
\Psi\left(r_{t}(e)\right) \leq(1-t) \Psi(g+h)+t \Psi(g+h(g)),
$$

but by definition of $h(g)$,

$$
\Psi(g+h(g)) \leq \Psi(g+h),
$$

so $\Psi\left(r_{t}(e)\right) \leq c$, and $r_{t}$ is the sought after homotopy inverse. It is obviously equivariant.

By $X^{-\infty}$ (resp. $Z^{-\infty}, E^{-\infty}, G^{-\infty}$ ) we mean some $X^{c}$ with $c<0,|c|$ large. We shall be more precise later, but it is already plain that computing $H_{S^{1}}^{*}\left(X, X^{-\infty}\right)$ or $H_{S^{\prime}}^{*}\left(Z, Z^{-\infty}\right)$ will yield the same result. We shall actually compute $H_{S 1}^{*}\left(X, X^{-\infty}\right)$.

The following preliminary lemma will prove useful:

Lemma 5.2. There is an $S^{1}$-invariant subspace $V$ of $X$ such that

$$
F \text { is bounded from below on } V \text {, }
$$

$F$ goes to minus infinity as $\|z\|$ goes to infinity on $V^{\perp}$,

(5.5) codim $V=d(K)$, where we define $d(K)$ to be $2 n([K T / 2 \pi]+1)$.

Proof. Define the quadratic form $A$ by

$$
A(z)=\int_{0}^{T}\left[(J \dot{z}-K z, z)+\frac{1}{(K+\varepsilon)}|J \dot{z}-K z|^{2}\right] d s,
$$

and take for $V^{\perp}$ the space generated by the eigenvectors associated with negative eigenvalues.

Let $z(t)=\sum_{-\infty}^{+\infty} \exp ((2 \pi / T) k J t) z_{k}$ be the Fourier decomposition of $z$; then we see that

$$
A(z)=\sum_{-\infty}^{+\infty} \frac{(2 \pi k-\varepsilon T)(2 n k+K T)}{(K+\varepsilon) T^{2}}\left|z_{k}\right|^{2},
$$

so that the codimension of $V$ is given by $2 n \#\{k \in \mathbf{Z} \mid-k T<2 \pi k<\varepsilon T\}=$ $d(K)$. This proves $(5.5)$.

Now by (2.9) of Proposition 2.7, we have

$$
\frac{\varepsilon}{2}|x|^{2}-C \leq H(x) \leq \frac{\varepsilon}{2}|x|^{2}+C
$$

for some constant $C$, so

$$
\frac{(K+\varepsilon)}{2}|x|^{2}-C \leq H_{K}(x) \leq \frac{(K+\varepsilon)}{2}|x|^{2}+C
$$


and

$$
\frac{1}{2(K+\varepsilon)}|y|^{2}-C \leq H_{K}^{*}(y) \leq \frac{1}{2(K+\varepsilon)}|y|^{2}+C,
$$

thus

$$
A(z)-C T \leq F(z) \leq A(z)+C T,
$$

and (5.3) and (5.4) are now straightforward.

The aim of this section is to prove

Proposition 5.7. If $c$ is large enough, then the inclusion $i: S\left(\rho, V^{\perp}\right) \rightarrow X^{-c}$ $\left(S\left(\rho, V^{\perp}\right)\right.$ is the intersection of $S(\rho)$, the sphere of radius $\rho$, and $\left.V^{\perp}\right)$ is an $S^{1}$-equivariant homotopy equivalence.

Let us first remark that by Proposition 5.1, the set of critical points of $F$ is compact, hence the set of critical levels is bounded. If $-b$ and $-c$ are less than the lower critical level, then assuming $b>c$, the inclusion $X^{-b} \hookrightarrow X^{-c}$ is an equivariant homotopy equivalence (e.h.e. for short).

We shall denote by $X^{-\infty}$ any of the set $X^{-c}$ with $c$ as above.

Definition 5.8. $p: X^{-\infty} \rightarrow S\left(\rho, V^{\perp}\right)$ is the composition of $\pi$, the orthogonal projection on $V^{\perp}$, and of the radial projection $r$ (which is well defined since $X^{-\infty} \cap V=\varnothing$ by (5.3)).

By the definition, $p \circ i=\mathrm{id}_{S\left(\rho, V^{\perp}\right)}$, our aim is to prove that $i \circ p$ is equivariantly homotopic to $\mathrm{id}_{X^{-\infty}}$; this implies Proposition 5.7.

Lemma 5.9. Set $\pi_{t}(z)=(1-t) z+t \pi(z)$. Given $c$, there exists $b$ such that $\pi_{t}\left(X^{-b}\right) \subset X^{-c}$ for $t \in[0,1]$.

Proof. Let us prove that if the lemma is true with $F$ replaced by $A$, then it is true for $F$.

By (5.6), we have

$$
A(z)-C T \leq F(z) \leq A(z)+C T,
$$

so if $A(z)<-b^{\prime}$ implies $A\left(\pi_{t}(z)\right)<-c^{\prime}$ then $F(z)<-b^{\prime}-C T=-b$ will imply

$$
F\left(\pi_{t}(z)\right)<-c^{\prime}+C T=-c .
$$

We then just have to prove the lemma with $A$ instead of $F$. But this is straightforward since we can write $A(z)=A\left(z_{+}\right)+A\left(z_{-}\right)$with $z_{+}=\pi(z)$, $z_{-}=z-\pi(z)$ and $A$ is positive on $V$, negative on $V^{\perp}$. Therefore

$$
\begin{aligned}
A\left(\pi_{t}(z)\right) & =A\left(t z_{+}\right)+A\left(z_{-}\right)=t^{2} A\left(z_{+}\right)+A\left(z_{-}\right) \\
& <A\left(z_{+}\right)+A\left(z_{-}\right)=A(z),
\end{aligned}
$$

whence our statement holds with $b^{\prime}=c^{\prime}$. 
Lemma 5.10. For $z \in V^{\perp}$, set $r_{\rho, t}(z)=(1-t) z+\operatorname{tr}_{\rho}(z)$ (we recall that $r_{p}$ is the radial projection of $V^{\perp}-\{0\}$ on $\left.S\left(\rho, V^{\perp}\right)\right)$. Given $d$, there exist $c$ and $\rho$ such that $X^{-c} \cap V=\varnothing$ and $r_{\rho, t}\left(X^{-c} \cap V^{\perp}\right) \subset X^{-d}$ for $t \in[0,1]$.

Proof. As for Lemma 5.9, if the lemma is true for $A$, it will be true for $F$. The case of $A$ is straightforward and left to the reader.

We can now proceed to the proof of Proposition 5.7.

Let $d$ be such that $-d$ is less than the smallest critical value of $F$; apply Lemma 5.10 to get a $c$. Given this $c$, Lemma 5.9 yields some $b$. Now if $F(z)<-b$, then $F\left(\pi_{t}(z)\right)<-c$, and $F\left(r_{\rho, t}(\pi(z))\right)<-d$.

The homotopy $\pi_{t}$ followed by $r_{\rho, t} \pi$ is then an equivariant homotopy of $X^{-b}$ in $X^{-d}$ from $\operatorname{id}_{X^{-h}}$ to $r_{\rho} \circ \pi=i \circ p$. Since $X^{-b}$ and $X^{-d}$ are e.h.e., this concludes our proof.

Corollary 5.11. $H_{S^{1}}^{q}\left(X, X^{-\infty}\right) \simeq H^{q-d(K)}\left(\mathbf{C} P^{\infty}\right)$ (which is one dimensional for all even $q$ 's larger than $d(K)$, and $\{0\}$ otherwise) .

Proof. It is clear by Proposition 5.7 that $H\left(X, X^{-\infty}\right) \simeq H\left(X, S\left(V^{\perp}\right)\right)$ where $S\left(V^{\perp}\right)$ denotes $S\left(1, V^{\perp}\right)$.

Now, according to Leray, there is a spectral sequence with an $E_{2}^{k, l}$ term equal to

$$
E_{2}^{k, l}=H^{k}\left(X, S\left(V^{\perp}\right)\right) \otimes H^{l}\left(\mathbf{C} P^{\infty}\right)
$$

and converging to $E_{\infty}^{k, l}$, where $\bigoplus_{k+l=q} E_{\infty}^{k, l}$ is the graded algebra of some filtration of $H_{S^{1}}^{q}\left(X, S\left(V^{\perp}\right)\right)$ (cf. [Sp, Chapter 9, §2]).

It is well known that $H^{k}\left(X, S\left(V^{\perp}\right)\right)$ is $\mathbf{Q}$ for $k=d(K)$ and 0 otherwise, hence $d_{2}=d_{3}=\cdots=0$, and this yields the result.

\section{Contribution of the solutions of $(\mathcal{N})$ to the Morse theory of $F_{K}$}

Let us point out that since $H$ is $C^{3}$ in $\mathbf{R}^{2 n}-\{0\}$, and the nonzero solutions of $(\mathscr{H})$ have their trajectories in $\mathbf{R}^{2 n}-\{0\}$, then according to (3.12) of Proposition 3.9, $\psi$ and $f$ are $C^{2}$ in a neighborhood of their nonzero critical points. According to Cambini's proof of the Morse lemma (cf. [Ca]), which requires only that the function be $C^{2}$, we can use Morse theory; that is, for $c \neq 0$ the groups $H_{S^{1}, *}\left(X^{c+\varepsilon}, X^{c-\varepsilon}\right)$ are computed in the usual way (cf. [B $\left.\mathrm{B}_{1}\right]$ ), provided all critical orbits at level $c$ are nondegenerate.

Let us recall how this works: by using a perturbation, we can always assume that $X^{c+\varepsilon}-X^{c-\varepsilon}$ contains a unique critical orbit of index $i$ and isotropy subgroup $\mathbf{Z}_{k}$. Then

$$
H_{S^{1}, *}\left(X^{c+\varepsilon}, X^{c-\varepsilon}\right) \simeq H_{*-i}\left(B \mathbf{Z}_{k}, \theta\right),
$$

where $\theta$ is the orientation rational bundle of the negative bundle of $\psi^{\prime \prime}$ (cf. [St] for the definition of homology with coefficients in a line bundle). 
We recall that if $\theta$ is the trivial bundle, that is, if $\nu^{-}$is orientable (this is always the case if $k$ is odd), then

$$
H_{*}\left(B \mathbf{Z}_{k} ; \theta\right)=H_{*}\left(B \mathbf{Z}_{k}, \mathbf{Q}\right)=\mathbf{Q}^{0}
$$

(i.e., $\mathbf{Q}$ in dimension zero, and 0 otherwise); if $\theta$ is not trivial, then $k$ is even and $\theta$ coincides with the orientation bundle of $B \mathbf{Z}_{2 k}, \mathbf{Q}_{t}$, and

$$
H_{*}\left(B \mathbf{Z}_{k}, \theta\right)=H_{*}\left(B \mathbf{Z}_{k}, \mathbf{Q}_{t}\right)=0 \text {. }
$$

Now, given a primitive solution of $(\mathcal{N})$, we consider the family of its iterates and wish to compute the contribution of this family to the Morse theory of $F$.

A first problem is due to the fact that the Morse index $\tilde{i}(K)$ of $x$, critical point of $F_{K}$ considered as a solution of $(\mathscr{H})$ depends on $K$. Fortunately, this dependence is quite simple according to the following lemma.

Lemma 6.4. We have $\tilde{i}(K)=2 n([K T / 2 \pi]+1)+i=d(K)+i$, where $i$ does not depend on $K$, but only on $H$.

Proof. See [V1, §5, Lemma 1], or [V2] (or [V3, the proof of Proposition 8]).

Definition 6.5. We shall call $i$ the index of $x$.

Remark 6.6. $i$ can be either positive, negative, or zero. This contrasts with the convex case, where $i$ is nonnegative.

Now let $y$ be a $\tau$-periodic solution $(\mathcal{N})$ and $k$ be an integer; then $y$ can also be considered as a $k \tau$-periodic solution of $(\mathscr{N})$. The corresponding solutions of $(\mathscr{H})$ shall be denoted by $x_{1}$ and $x_{k}$, respectively, and $x_{k}$ is $T / k$-periodic. Then if $i_{1}$ is the index of $x_{1}$, we write $i_{k}$ for the index of $x_{k}$.

Now as in [B2 and E1] we shall relate $i_{k}$ with $i_{1}$ and the Poincare map of $x_{1}$, that is,

Definition 6.7. If $\phi_{s}$ is the flow of our Hamiltonian vector field, and $x_{1}$ is a solution of $(\mathscr{H})$ (i.e., $\left.\phi_{T}\left(x_{1}\right)=x_{1}\right)$, then we define the (linear) Poincare map of $x_{1}$ to be $R=d \phi_{T}\left(x_{1}(0)\right)$.

We shall assume for all primitive orbits that the Poincare map $R$ satisfies the following generic conditions:

The eigenspace and characteristic space of $R$ corresponding to the eigenvalue 1 are respectively one dimensional and two dimensional.

$$
\begin{aligned}
& \text { If } \omega_{1}=e^{2 i \pi \theta_{1}}, \ldots, \omega_{m}=e^{2 i \pi \theta_{m}}, \bar{\omega}_{1}, \ldots, \bar{\omega}_{m} \text { are the other } \\
& \text { eigenvalues of } R \text { on the unit circle, then the } \theta_{i} \text { 's are irrationals. }
\end{aligned}
$$

According to [Ta], the set of hypersurfaces for which (6.8) and (6.9) hold for all periodic solutions is a Baire set.

Let us recall that if $\omega$ is a eigenvalue of $R$ on the unit circle, then $\omega$ is said to be of Krein type $(p, q)$ if on $\operatorname{ker}(R-\omega I) \subset \mathbf{C}^{2 n}$ the hermitian form $i(J z, z)$ has signature $(p, q)$ (cf. [Y-S]). 
We can now state

Definition 6.10. Given $x_{1}$ solution of $(\mathscr{H})$ with Poincaré map $R$, we define a function $j: S^{1}-\left\{\omega_{1}, \ldots, \omega_{m}, \bar{\omega}_{1}, \ldots, \bar{\omega}_{m}\right\} \rightarrow \mathbf{Z}$ by setting

$$
j(1)=i_{1}, \quad \lim _{\varepsilon \rightarrow 0} j\left(e^{i \varepsilon}\right)=i_{1}+n+1,
$$

$j$ is locally constant ,

where $\left(p_{l}, q_{l}\right)$ is the Krein type of $\omega_{l}$.

Proposition 6.14. We have the following.

$$
i_{k}=\sum_{\omega^{k}=1} j(\omega)
$$

or

$$
i_{k}=i_{1}+(k-1) j(-1)-2 \sum_{l=1}^{m} \Delta_{j}\left(\omega_{l}\right)\left[k \theta_{l}\right]
$$

hence

(i) there are constants $c, c^{\prime}$ such that

$$
k \hat{\imath}-c \leq i_{k} \leq k \hat{\imath}+c^{\prime}
$$

where $\hat{\imath}=(1 / 2 \pi) \int_{S^{1}} j(\omega) d \omega$

(ii) $i_{k}=i_{1}+(k-1) j(-1)$ modulo 2 .

Proof. This is a generalization of the results of [E1, $\S \S 4$ and 5], and was carried out in [V1, V2]. The idea of the formula stated in Proposition 6.14 goes back to Bott (cf. [B2]) for the case of geodesics.

Now let $x_{1}$ be a primitive solution; the following tells us whether the $x_{2 k}$ are orientable or not.

Proposition 6.15. The $x_{2 k}$ are all orientable if $j(-1)$ is even, all unorientable if $j(-1)$ is odd.

Proof. $x_{2 k}$ is a $T$-periodic solution of $(\mathscr{H})$ which is in fact $T / 2 k$-periodic. The negative bundle of $x_{2 k}$ is endowed with a $\mathbf{Z} / 2 k \mathbf{Z}$-action, the generator of this group corresponding to the map

$$
r_{T / 2 k}: u(\cdot) \rightarrow u(T / 2 k+\cdot) .
$$

Notice that if $F$ is the fiber of the negative bundle over $x_{2 k}$, we can identify the total negative bundle to $[0, T / 2 k] \times F / \sim$ where

$$
(0, f) \sim\left(T / 2 k, r_{T / 2 k}(f)\right) .
$$

Thus the bundle is orientable if and only if $r_{T / 2 k}$ is orientation preserving.

Since $\left(r_{T / 2 k}\right)^{2 k}=\mathrm{Id}$, because all $u$ 's are $T$-periodic, $F$ splits as a direct sum of subspaces on which $r_{T / 2 k}$ is respectively a rotation of angle $\theta \notin \mathbf{Z} \pi$, 
the identity, and minus identity. Obviously, $r_{T / 2 k}$ will then be orientation preserving if and only if the dimension of this last subspace is even, that is, if $\operatorname{dim} \operatorname{ker}\left(r_{T / 2 k}+I\right)$ is even, or else if $\operatorname{dim} \operatorname{ker}\left(r_{T / 2 k}^{2}-I\right)-\operatorname{dim} \operatorname{ker}\left(r_{T / 2 k}-I\right)$ is even.

But the vectors of $\operatorname{ker}\left(r_{T / 2 k}-I\right)$ are the $T / 2 k$-periodic elements of the negative bundle of $x_{2 k}$, that is, the iterates of the vectors in the negative bundle of $x_{1}$, so $\operatorname{dim} \operatorname{ker}\left(x_{T / 2 k}-I\right)=i_{1}(K)$. By the same argument,

$$
\operatorname{dim} \operatorname{ker}\left(\left(r_{T / 2 k}\right)^{2}-I\right)=i_{2}(K),
$$

and since $i_{2}(K)-i_{1}(K)=i_{2}-i_{1}=j(-1)$, this completes our proof.

Now recall that $H$ depends on $a$ (cf. Proposition 2.7) and $x_{k}$ is a critical circle of $F_{K}$ if and only if $k \tau \leq a T / 2$, where $\tau$ is the period of the solution of $(\mathscr{N})$ corresponding to $x_{1}$.

We assume that $x_{1}$ is a primitive solution, and we compute the contribution of the $x_{k}$, for $k \tau \leq a T / 2$, to the Morse series of $F_{K}:$ this is given by

$$
t^{d(K)} \sum_{\substack{k \tau \leq a T / 2 \\ x_{k} \text { orientable }}} t^{i_{k}}=M_{K, a}\left(x_{1} ; t\right) .
$$

We set $M_{a}\left(x_{1} ; t\right)=t^{-d(K)} M_{K, a}\left(x_{1} ; t\right)$ and $M_{a}(t)=\sum M_{a}\left(x_{1} ; t\right)$, the sum being extended over all primitive solutions of $(\mathscr{N})$.

Notice that for a generic hypersurface, there are only finitely many periodic orbits of period smaller than $a T / 2$, so our sum is bounded for any given $a$.

Moreover, set $M_{a}(t)=\sum_{l \mathrm{Z}} m_{l}(a) t^{l}$; we claim that for $|l| \geq 2 n^{2}$ and if there are only finitely many periodic orbits, then $m_{l}=\lim _{a \rightarrow+\infty} m_{l}(a)$ is finite.

Indeed, if $\hat{\imath}\left(x_{1}\right) \neq 0$, then $\left|i_{k}\right|$ goes to infinity with $k$ (cf. 6.14(i)) hence the contribution of the $x_{k}$ to $m_{l}(a)$ is bounded. On the other hand, if $\hat{\imath}=0, j$ takes positive as well as negative values. By definition (cf. (6.13)) the variation of $j$ at $\omega_{l}$ has absolute value less than $n$ and it is easily checked that (using 6.14)

$$
\left|i_{k}-k \hat{\imath}\right| \leq \sum_{l=1}^{m}\left|\Delta j\left(\omega_{l}\right)\right| \leq n m \leq n^{2} .
$$

So if $\hat{\imath}=0,\left|i_{k}\right| \leq n^{2}$ and thus does not contribute to $m_{l}(a)$. This proves our claim.

We can set

\section{Definition 6.17.}

$$
\chi^{+}=\lim _{N \rightarrow+\infty} \frac{1}{N} \sum_{l=2 n^{2}}^{N}(-1)^{l} m_{l}, \quad \chi^{-}=\lim _{N \rightarrow+\infty} \frac{1}{N} \sum_{l=2 n^{2}}^{N}(-1)^{l} m_{-l} .
$$

Remark. $\chi^{+}$and $\chi^{-}$are some sort of average Euler characteristics; indeed we shall show that their value is an invariant.

We now prove the main statement of this section. 
Proposition 6.18. The contribution of a primitive solution of $(\mathcal{N})$ and its iterates to $\chi^{+}$(resp. $\left.\chi^{-}\right)$is given by

(i) If $j(-1)$ is even, $(-1)^{i_{1}} / \hat{\imath}$ if $\hat{\imath}>0$ (resp. $\hat{\imath}<0$ ) and zero otherwise.

(ii) If $j(-1)$ is odd, $(-1)^{i_{1}} / \hat{\imath}$ if $\hat{\imath}>0$ (resp. $\hat{\imath}<0$ ) and zero otherwise.

Proof. We shall only consider the case $\hat{\imath}>0$; the other case is similar.

If $j(-1)$ is even, all the $x_{k}$ are orientable, so the contribution of our family to $\chi^{+}$is given by $\lim _{N \rightarrow+\infty}(1 / N) \sum_{2 n^{2} \leq i_{k} \leq N}(-1)^{i_{k}}$.

By 6.14(ii), $i_{k}=i_{1}$ modulo 2 , so the sum reduces to $(-1)^{i_{1}} \#\left\{k \mid 2 n^{2} \leq i_{k} \leq\right.$ $N$, and using $6.14(\mathrm{i})$, this is asymptotically $(-1)^{i_{1}} N / \hat{\imath}$, hence our result.

It is easy to check that the contribution to $\chi^{-}$is zero since $i_{k} \geq-2 n^{2}$.

Finally, if $j(-1)$ is odd, only the $x_{2 k+1}$ are orientable, so the contribution to $\chi^{+}$is given by

$$
\lim _{N \rightarrow+\infty} \frac{1}{N} \sum_{2 n^{2} \leq 2 k+1 \leq N}(-1)^{i_{2 k+1}}
$$

and since $i_{2 k+1}=i_{1} \bmod 2$ our sum is equal to $(-1)^{i_{1}} \#\left\{k \mid 2 n^{2} \leq i_{2 k+1} \leq N\right\}$, which is asymptotically $(-1)^{i_{1}} N / 2 \hat{\imath}$, yielding our result.

\section{CONTRIBUtion OF THE ORIGIN}

By contribution of the origin, we mean $H_{S^{1}, *}\left(X^{\ell}, X^{-\varepsilon}\right)$. Since it is easily shown that there are no other critical points than the origin in $\left(X^{\ell} \backslash X^{-\varepsilon}\right)$, our terminology is appropriate.

First, let us show that this contribution depends only on the restriction of $H$ to a neighborhood of the origin, and thus we can assume $H$ to be homogeneous of degree two everywhere.

We shall consider the case where $(\mathscr{N})$ has no periodic solution of period $a T / 2$ (if the set of periods of solutions of $(\mathscr{N})$ is discrete, we can always pick such an $a$; otherwise there are obviously infinitely many primitive solutions), so that the origin is an isolated critical point of $f$. Then in Conley's terminology (cf. [Co]) any neighborhood of the origin is an isolated invariant set, hence the contribution of the origin depends only on the restriction of $f$ to such a neighborhood. But if $x \in X$ is small, then $\left\{x(t) \mid t \in S^{1}\right\}$ is in a neighborhood of 0 in $\mathbf{R}^{2 n}$, and this proves our assertion.

From now on, we assume $H$ to be homogeneous of degree two and everything shall refer to this new $H: f, \psi, X^{c}, E^{c}, \ldots$. Also the contribution of the origin is given by $H_{S^{1}, *}\left(X^{\varepsilon}, X^{-\varepsilon}\right)$ for any positive $\varepsilon$.

The aim of this section is to prove

Theorem 7.1. Let $I$ be an interval of $\mathbf{Z}$ such that no solution of $(\mathscr{N})$ with period larger than $a T / 2$ has index in $I$. Then if $\mathbf{F}=\mathbf{Q}$, we have

$$
H_{S^{1}, k+d(K)}\left(X^{\ell}(a, K), X^{-\varepsilon}(a, K)\right)=0 \text { for } k \in \stackrel{\circ}{I}
$$

(where $\stackrel{\circ}{I}$ is $I$ minus its endpoints). 
We denote $X^{\varepsilon}$ by $X^{\varepsilon}(a, K)$ to remind the reader that it depends on both $a$ and $K$.

The proof of Theorem 7.1 is done in several steps, which we now shortly describe.

Preliminary remark. By homogeneity of $F_{a, K}$, we can replace $\left(X^{\varepsilon}(a, K)\right.$, $\left.X^{-\varepsilon}(a, K)\right)$ by $\left(X, X^{-}(a, K)\right)$ where

$$
X^{-}(a, K)=\left\{x \in X \mid F_{a, K}(x)<0\right\} .
$$

1st step: We show that there is an isomorphism

$$
H_{S^{1}, k+d(K)}\left(X, X^{-}(a, K)\right) \rightarrow H_{S^{1}, k+d\left(K^{\prime}\right)}\left(X, X^{-}\left(a, K^{\prime}\right)\right),
$$

given by the geometric construction that we shall now describe. First of all, we claim that if $Y$ is a space with an $S^{1}$-action, any class in $H_{S^{1}, *}(Y)$ is in the image of some $\phi_{*}: H_{S^{1, *}}(A) \rightarrow H_{S^{1}, *}(Y)$, where $\phi: A \rightarrow Y$ is an equivariant map between the compact set $A$ endowed with an $S^{1}$-action and $Y$. In order to check our claim, we first construct $\bar{\phi}: \bar{A} \rightarrow Y \times E S^{1} / S^{1}$ such that $\bar{A}$ is compact and our class is in the image of $H_{*}(\bar{A})$ in $H_{*}\left(Y \times E S^{1} / S^{1}\right) \simeq H_{S^{1}, *}(Y)$. Then $Y \times E S^{1}$ is an $S^{1}$-bundle over $Y \times E S^{1} / S^{1}$; denote by $A$ its pullback by $\bar{\phi}$. Then $\bar{\phi}$ is covered by an equivariant map $\tilde{\phi}: A \rightarrow Y \times E S^{1}$. Since the projection $Y \times E S^{1} \rightarrow Y$ induces a canonical isomorphism $H_{S^{1}, *}\left(Y \times E S^{1}\right) \rightarrow H_{S^{1}, *}(Y)$, if we take for $\phi$ the composition of $\tilde{\phi}$ and the above mentioned projection, we are done.

Obviously, the same holds for relative homology. Consider then a map $\gamma$ : $\left(\Gamma_{1}, \Gamma_{2}\right) \rightarrow\left(X, X^{-}(a, K)\right)$ where $\left(\Gamma_{1}, \Gamma_{2}\right)$ is a compact pair of spaces with an $S^{1}$-action, inducing $\gamma_{*}: H_{S^{1}, *}\left(\Gamma_{1}, \Gamma_{2}\right) \rightarrow H_{S^{1}, *}\left(X, X^{-}(a, K)\right)$. Set $D_{R}\left(K, K^{\prime}\right)$ $=\bigoplus_{K \leq L \leq K^{\prime}} D_{R}(L)$, and for $L$ in $(2 \pi / T) \mathbf{Z}$, we set

$$
D_{R}(L)=\left\{\exp (-J L t) x \mid x \in \mathbf{R}^{2 n},\|x\| \leq R\right\},
$$

and define $\gamma^{\prime}=\gamma \oplus D_{R}\left(K, K^{\prime}\right)$ to be the map

$$
\gamma^{\prime}:\left(\Gamma_{1}, \Gamma_{2}\right) \times\left(D_{R}\left(K, K^{\prime}\right), \partial D_{R}\left(K, K^{\prime}\right)\right) \rightarrow\left(X, X^{-}\left(a, K^{\prime}\right)\right)
$$

(note: $(A, B) \times(C, D)$ means $(A \times C, B \times C \cup A \times D))$ given by $\gamma^{\prime}(x, u)=$ $\gamma(x)+u$.

Remark. Let us mention that the assertion

$$
\gamma^{\prime}\left(\Gamma_{2} \times D_{R}\left(K, K^{\prime}\right), \Gamma_{1} \times \partial D_{R}\left(K, K^{\prime}\right)\right) \subset X^{-}(a, K)
$$

is not trivial and holds only for $R$ large enough, which we shall assume henceforth. The proof that this inclusion holds will appear later in this section.

As we shall see, there is a natural isomorphism (induced by the Thom isomorphism of some fibration)

$$
H_{S^{1}, k+d(K)}\left(\Gamma_{1}, \Gamma_{2}\right) \rightarrow H_{S^{1}, k+d\left(K^{\prime}\right)}\left(\Gamma_{1}, \Gamma_{2}\right) \times\left(D_{R}\left(K, K^{\prime}\right), \partial D_{R}\left(K, K^{\prime}\right)\right),
$$


and we claim that $\gamma_{*}, \gamma_{*}^{\prime},(7.2)$, and (7.3) are the edges of a commutative diagram.

2nd step: Since $X\left(a, K^{\prime}\right) \subset X^{-}\left(a^{\prime}, K^{\prime}\right)$ for $a^{\prime}>a$ there is a map

$$
H_{S^{\prime}, k+d(K)}\left(X, X^{-}\left(a, K^{\prime}\right)\right) \rightarrow H_{S^{\prime}, k+d\left(K^{\prime}\right)}\left(X, X^{-}\left(a^{\prime}, K^{\prime}\right)\right)
$$

which, compared with (7.2), yields a map

$$
H_{S^{1}, k+d(K)}\left(X, X^{-}(a, K)\right) \rightarrow H_{S^{1}, k+d\left(K^{\prime}\right)}\left(X, X^{-}\left(a^{\prime}, K^{\prime}\right)\right) .
$$

We prove that if $a^{\prime}$ and $K^{\prime}$ are large enough, the map (7.4) is zero. This is achieved by showing that if $\gamma$ represents some class in $H_{S^{1}, k+d(K)}\left(X, X^{-}(a, K)\right)$, then $\gamma \oplus D_{R}\left(K, K^{\prime}\right)$ has its image contained in $X^{-}\left(a^{\prime}, K^{\prime}\right)$ for $a^{\prime}$ large enough.

3rd step: The map $H_{S^{1}, k+d\left(K^{\prime}\right)}\left(X, X^{-}\left(a, K^{\prime}\right)\right) \rightarrow H_{S^{1}, k+d\left(K^{\prime}\right)}\left(X, X^{-}\left(a^{\prime}, K^{\prime}\right)\right)$ is an isomorphism for $k$ in $\stackrel{\circ}{I}$, and $a$ such that any periodic orbit of $(\mathscr{N})$ with period larger than $a T / 2$ has period in $\stackrel{\circ}{I}$. This is proved by showing that $X^{-}\left(a^{\prime}, K^{\prime}\right)$ has the equivariant homotopy type of $X^{-}\left(a, K^{\prime}\right)$ with equivariant cells attached, corresponding to the solutions of $(\mathcal{N})$ with period in $\left[a T / 2, a^{\prime} T / 2\right]$. From this and step 1 , we infer that (7.4) is an isomorphism for $k$ in $\stackrel{\circ}{I}$. On the other hand, according to step 2, this map is zero, hence $H_{S^{1}, k+d(K)}\left(X, X^{-}(a, K)\right)$ is zero for $k$ in $\stackrel{\circ}{I}$.

Let us now give the complete proofs.

Preliminary remark. We want to prove that the inclusion

$$
\left(X^{\varepsilon}(a, K), X^{-\varepsilon}(a, K)\right) \hookrightarrow\left(X, X^{-}(a, K)\right)
$$

is a weak equivariant homotopy equivalence (e.h.e. in the sequel). This follows from the fact that $F_{a, K}$ satisfies the Palais-Smale condition and has zero as its unique critical level, so that $X^{\varepsilon}(a, K) \rightarrow X$ is an e.h.e., and $X^{-\varepsilon}(a, K) \hookrightarrow$ $X^{-\varepsilon^{\prime}}(a, K)$ also, for $\varepsilon^{\prime}<\varepsilon$. From this last assertion, $X^{-\varepsilon}(a, K) \hookrightarrow X^{-}(a, K)$ $=\bigcup_{\varepsilon^{\prime}>0} X^{-\varepsilon^{\prime}}(a, K)$ is an e.h.e., hence our result.

Step 1. This is the longest and most intricate part of our proof. We use induction, together with the two following special cases of step 1:

(a) $d(K)=d\left(K^{\prime}\right)$;

(b) $K<K_{0}<K^{\prime}$ with $K_{0}-K, K^{\prime}-K_{0}$ small, and $K_{0} T / 2 \pi$ is an integer. It is then clear that (a) and (b) imply the general case.

To begin with, we consider case (a). An easy but tiresome computation shows

$$
\frac{\partial}{\partial K} F_{a, K}(x)=-\frac{1}{2}\left\|x-\nabla H_{K}^{*}(-J \dot{x}+K x)\right\|^{2} .
$$

Indeed write

$$
F_{a, K}(x)=\int_{0}^{T}\left[\left(\frac{1}{2} J \dot{x}-K x, x\right)+H_{k}^{*}(-J \dot{x}+K x)\right] d t,
$$


so that

$$
\frac{\partial}{\partial K} F_{a, K}(x)=-\frac{1}{2}\|x\|^{2}+\int_{0}^{T} \frac{\partial}{\partial K}\left(H_{K}^{*}(-J \dot{x}+K x)\right) d t .
$$

We first compute $\partial H_{K}^{*}(u) / \partial K$ : set $v_{K}=H_{K}^{*}(u)$ (i.e., $\nabla H_{K}\left(v_{K}\right)=u$ ); then

$$
H_{K}^{*}(u)=\left(v_{K}, u\right)-H_{K}\left(v_{K}\right)
$$

and

$$
\frac{\partial}{\partial K} H_{K}^{*}(u)=\left(\frac{\partial}{\partial K} v_{K}, u\right)-\left(\frac{\partial}{\partial K} H_{K}\right)\left(v_{K}\right)-\left(\nabla H_{K}\left(v_{K}\right), \frac{\partial}{\partial K} v_{K}\right) .
$$

Since $\partial H_{K}(x) / \partial K=\frac{1}{2}|x|^{2}$, we get

$$
\frac{\partial}{\partial K} H_{K}^{*}(u)=\left(\frac{\partial}{\partial K} v_{K}, u\right)-\frac{1}{2}\left|v_{K}\right|^{2}-\left(u, \frac{\partial}{\partial K} v_{K}\right)=-\frac{1}{2}\left|\nabla H_{K}^{*}(u)\right|^{2} .
$$

Now

$$
\frac{\partial}{\partial K} H_{K}^{*}(-J \dot{x}+K x)=-\frac{1}{2}\left|\nabla H_{K}^{*}(-J \dot{x}+K x)\right|^{2}+\left(\nabla H_{K}^{*}(-J \dot{x}+K x), x\right),
$$

whence (7.5).

From (7.5), we see that $\partial F_{a, K}(x) / \partial K$ is negative (it can be zero only if $x=\nabla H_{K}^{*}(-J \dot{x}+K x)$ and $x$ would yield an $a T / 2$ periodic solution of $(\mathscr{N})$, which was excluded). From (7.5) we use the fact that $X^{-}(a, K)$ is contained in $X^{-}\left(a, K^{\prime}\right)$ if $K^{\prime}>K$.

We now prove that the sets $X^{-}(a, L)$ for $L$ in $\left[K, K^{\prime}\right]$ are all isotopic.

Since the $F_{a, L}$ are homogeneous, the $X^{-}(a, L)$ are stable by dilation, so it is enough to prove that the $X^{-}(a, L) \cap S$ are isotopic, where $S$ is the unit sphere in $X$.

The restriction of $F_{a, L}$ to $S$ has zero as regular value since if $\nabla F_{a, L}(x)=\lambda x$ then $2 F_{a, L}(x)=\left(\nabla F_{a, L}(x), x\right)=\lambda\|x\|^{2}$, implying that a critical point of level zero is a critical point of the unconstrained function. But, by assumption, the only such critical point is the origin, which is not on $S$.

Now by Proposition 1 of Appendix 1, this implies that $X^{-}\left(a, K^{\prime}\right)$ is equivariantly isotopic to $X^{-}(a, K)$, hence the inclusion $X^{-}(a, K) \hookrightarrow X^{-}\left(a, K^{\prime}\right)$ is an e.h.e. This concludes the proof of (a).

We now consider case (b). Let $C\left(K_{0}\right)$ denote the orthogonal of $D_{\infty}\left(K_{0}\right)$ in $X$. Let us remark that in the direction of $D_{\infty}\left(K_{0}\right), F_{K}$ (resp. $\left.F_{K^{\prime}}\right)$ is convex (resp. concave); that is, to be precise, for all $x$ in $X, h$ in $D_{\infty}\left(K_{0}\right)$,

$$
\begin{gathered}
\left(F_{L}^{\prime \prime}(x) h, h\right) \geq C\left(K_{0}-L\right)\|h\|^{2} \quad \text { if } L \leq K_{0}, \\
\left(F_{L}^{\prime \prime}(x) h, h\right) \leq-C\left(L-K_{0}\right)\|h\|^{2} \quad \text { if } L \geq K_{0},
\end{gathered}
$$

where $C$ is independent of $L, x, h$ and $F_{L}^{\prime \prime}(x)$ is defined as in $\S 5$.

The proof of $(7.6),(7.7)$ is just computational: if $h \in D_{\infty}\left(K_{0}\right),-J \dot{h}+L h=$ $-J \dot{h}+K_{0} h+\left(L-K_{0}\right) h=\left(L-K_{0}\right) h$; hence

$$
\left(F_{L}^{\prime \prime}(x) h, h\right)=\int_{0}^{T}\left[-\frac{1}{2}\left(L-K_{0}\right)|h|^{2}+\left(L-K_{0}\right)^{2}\left(H_{L}^{* \prime \prime}(-J \dot{x}+L x) h, h\right)\right] d s \text {. }
$$


Since $H_{L}^{* \prime \prime}$ is uniformly bounded, we have that for $\left(L-K_{0}\right)$ small enough, the linear term in $\left(L-K_{0}\right)$ dominates the quadratic one, yielding (7.6) and (7.7).

Before we state Lemma 7.9, the crucial step of our proof, we need a definition.

Definition 7.8. For $Y \subset C\left(K_{0}\right)$, we denote by $Y \times D\left(K_{0}\right)$ the union of $y \oplus$ $D_{\phi(y)}\left(K_{0}\right)$ for $y \in Y$ where $\phi: C\left(K_{0}\right) \rightarrow R_{+}^{*}$ is some continuous function on $C\left(K_{0}\right)$.

Our next lemma is

Lemma 7.9. The following inclusions are e.h.e.:

$$
\begin{gathered}
X^{-}(a, L) \rightarrow X^{-}\left(a, K_{0}\right) \quad \text { for } L<K_{0}, \\
X^{-}\left(a, K_{0}\right) \cup \complement \overline{C\left(K_{0}\right) \times D\left(K_{0}\right)} \rightarrow X^{-}(a, L) \text { for } L>K_{0} .
\end{gathered}
$$

Remark. If $L>K_{0}$, then for $y \in C\left(K_{0}\right), h \rightarrow F_{a, L}(y+h)$ defined on $D_{\infty}\left(K_{0}\right)$ is strictly concave, according to (7.7). Thus, if $\|h\|$ is large enough, $y+h$ is in $X^{-}(a, L)$, implying that $(7.11)$ is indeed an inclusion.

Proof of the Lemma. In this proof, $a$ is fixed, so we write $F_{L}$ for $F_{a, L}$. By strict convexity (resp. concavity) of $F_{L}$, for $L<K_{0}$ (resp. $L>K_{0}$ ) there is a map

$$
C\left(K_{0}\right) \rightarrow D_{\infty}\left(K_{0}\right), \quad y \rightarrow z_{L}(y),
$$

uniquely defined by the relation $\nabla F_{L}\left(y+z_{L}(y)\right) \in C\left(K_{0}\right)$; that is, $z_{L}(y)$ achieves the minimum of $F_{L}(g+h)$ for $h$ in $D_{\infty}\left(K_{0}\right)$.

Then we claim that

$$
\begin{gathered}
\left|F_{L}(x)-F_{K_{0}}(x)\right| \leq C\left|L-K_{0}\right|\|x\|_{H^{1}}, \\
\left\|\nabla F_{L}(x)-\nabla F_{K_{0}}(x)\right\|_{H^{1}} \leq C\left|L-K_{0}\right|\|x\|_{H^{1}} .
\end{gathered}
$$

The inequality (7.12) follows immediately from (7.5). To prove (7.13), take the gradient of (7.5), yielding $\frac{\partial}{\partial L}\left(\nabla F_{L}(x), y\right)=-\left\langle x-\nabla H_{L}^{*}(-J \dot{x}+L x), y-H_{L}^{* \prime \prime}(-J \dot{x}+L x)(-J \dot{y}+L y)\right\rangle ;$ since $H_{L}^{* \prime \prime}$ is bounded, this yields

$$
\left|\frac{\partial}{\partial L} \nabla F_{L}(x) \cdot y\right| \leq C\|x\|_{H^{1}}\|y\|_{H^{\prime}},
$$

hence

which yields (7.13) by integration.

$$
\left\|\frac{\partial}{\partial L} \nabla F_{L}(x)\right\|_{H^{1}} \leq C\|x\|_{H^{1}},
$$

Now for $y \in C\left(K_{0}\right)$, set $\widetilde{F}_{L}(y)=F_{L}\left(y+z_{L}(y)\right)$, and notice that

$$
\nabla \tilde{F}_{L}(y)=\nabla F_{L}\left(y+z_{L}(y)\right)\left(I+z_{L}^{\prime}(y)\right)=\nabla F_{L}\left(y+z_{L}(y)\right),
$$

because $\nabla F_{L}\left(y+z_{L}(y)\right)$ is zero on the image of $z_{L}^{\prime}(y)$ which is contained in $D_{\infty}\left(K_{0}\right)$. 
As we shall prove later, the following holds:

$$
\left|z_{L}(y)\right| \leq C\|y\|_{H^{1}},
$$

with $C$ independent of $L$.

Using (7.12), (7.13), and (7.14), we infer

$$
\begin{gathered}
\left|\widetilde{F}_{L}(y)-F_{K_{0}}\left(y+z_{L}(y)\right)\right| \leq C\left|L-K_{0}\right|\|y\|_{H^{1}}^{2}, \\
\left\|\nabla \widetilde{F}_{L}(y)-\nabla F_{K_{0}}\left(y+z_{L}(y)\right)|\leq C| L-K_{0} \mid\right\| y \|_{H^{1}}^{2} .
\end{gathered}
$$

Since $F_{K_{0}}$ is constant in the $D_{\infty}\left(K_{0}\right)$ direction, the last two inequalities can be written

$$
\begin{gathered}
\left|\widetilde{F}_{L}(y)-F_{K_{0}}(y)\right| \leq C\left|L-K_{0}\right|\|y\|_{H^{1}}^{2}, \\
\left\|\nabla \widetilde{F}_{L}(y)-\nabla F_{K_{0}}(y)\right\|_{H^{1}} \leq C \mid L-K_{0}\|y\|_{H^{1}}^{2} .
\end{gathered}
$$

Now $\widetilde{F}_{L}$ is homogeneous, because $F_{L}$ is, and for $\left|L-K_{0}\right|$ small, the restriction of $\widetilde{F}_{L}$ to $S \cap C\left(K_{0}\right)$ is $C^{1}$ close to $F_{K_{0}}$. Since the restriction of $F_{K_{0}}$ to $S \cap C\left(K_{0}\right)$ has no critical points on level zero (as before, such a critical point would yield a critical point of the unconstrained function $F_{K_{0}}$, whence we would have an $a T / 2$ periodic solution of $(\mathscr{N})$, contradicting our assumptions), using Appendix 1, Proposition 1(i), and the homogeneity of $\widetilde{F}_{L}$, we see that the map

$$
\tilde{X}^{-}(a, L) \rightarrow \tilde{X}^{-}\left(a, K_{0}\right)
$$

-where we define $\tilde{X}^{-}(a, L)$ to be $\left\{y \in C\left(K_{0}\right) \mid \widetilde{F}_{L}(y)<0\right\}$-is an e.h.e.

Now if $L \leq K_{0}, X^{-}(a, L)$ is a fiber space over $\tilde{X}^{-}(a, L)$ with convex (hence contractible) fiber: from the convexity of $F_{L}$ in the $D_{\infty}\left(K_{0}\right)$ direction, we infer that $F_{L}\left(y_{+} z_{L}(y)+h\right)<0$ implies $F_{L}\left(y+z_{L}(y)\right)=\widetilde{F}_{L}(y)<0$ and $\left\{h \in D_{\infty}\left(K_{0}\right) \mid F_{L}\left(y+z_{L}(y)+h\right)<0\right\}$ is convex and nonempty if and only if $\widetilde{F}_{L}(y)<0$.

As a result we have the following commutative diagram:

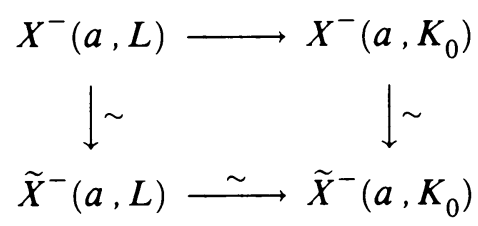

where " " denotes an e.h.e.

From this diagram, we conclude that the inclusion $X^{-}(a, L) \rightarrow X^{-}\left(a, K_{0}\right)$ is also an e.h.e. if $L<K_{0}$, thus proving the first part of the lemma.

We now assume $L>K_{0}$, and consider for $y \in C\left(K_{0}\right)$ the set $N_{y}=\{h \in$ $\left.D_{\infty}\left(K_{0}\right) \mid F_{L}\left(y+z_{L}(y)+h\right)<0\right\}$. Since $F_{L}$ is concave, and the maximum of $F_{L}\left(y+z_{L}(y)+h\right)$ on $D_{\infty}\left(K_{0}\right)$ is achieved only when $h=0, N_{y}$ is $D_{\infty}\left(K_{0}\right)$ if 
$y \in \tilde{X}^{-}(a, L)$ and the complement of a nonempty convex set otherwise. There is then an inclusion

$$
\tilde{X}^{-}(a, L) \times D_{\infty}\left(K_{0}\right) \cup \complement \overline{C\left(K_{0}\right) \times D\left(K_{0}\right)} \rightarrow X^{-}(a, L)
$$

(where the $\phi$ of Definition 7.8 is defined so that $C \overline{D_{\phi(y)}\left(K_{0}\right)}$ is contained in $N_{y}$ ); let us show that this is an e.h.e.

It is easily checked that there is an e.h.e.

$\tilde{X}^{-\varepsilon / 2}(0, L) \times D_{\infty}\left(K_{0}\right) \cup X^{-\varepsilon}(a, L) \rightarrow \tilde{X}^{-}(a, L) \times D_{\infty}\left(K_{0}\right) \cup \complement \overline{C\left(K_{0}\right) \times D\left(K_{0}\right)}$

induced by the inclusion. In fact, we can identify $X^{-\varepsilon}(a, L) \backslash \tilde{X}^{-\varepsilon / 2}(a, L) \times$ $D_{\infty}\left(K_{0}\right)$ to

$$
\left\lceil\overline{C\left(K_{0}\right) \times D\left(K_{0}\right)} \backslash \widetilde{X}^{-\varepsilon / 2}(a, L) \times D_{\infty}\left(K_{0}\right),\right.
$$

since if $F_{a, L}(y+h)<-\varepsilon$ but $F_{a, L}\left(y+z_{L}(y)\right)>-\varepsilon / 2$ by the concavity of $F_{a, L}$ in the $D_{\infty}\left(K_{0}\right)$ direction, $h$ is in the complement of a convex set of nonempty interior, which we can identify with the fiber of

$$
\left\lceil\overline{C\left(K_{0}\right) \times D\left(K_{0}\right)} \backslash \tilde{X}^{-\varepsilon / 2}(a, L) \times D_{\infty}\left(K_{0}\right) .\right.
$$

Therefore, we can identify $\tilde{X}^{-\varepsilon / 2}(a, L) \times D_{\infty}\left(K_{0}\right) \cup X^{-\varepsilon}(a, L)$ and $\tilde{X}^{-\varepsilon / 2}(a, L)$ $\times D_{\infty}\left(K_{0}\right) \cup C \overline{C\left(K_{0}\right) \times D\left(K_{0}\right)}$. Now since $\tilde{X}^{-}(a, L)$ and $\tilde{X}^{-\varepsilon / 2}(a, L)$ are e.h.e., our statement is proved.

We then have to prove that the inclusion

$$
\tilde{X}^{-\varepsilon / 2}(a, L) \times D_{\infty}\left(K_{0}\right) \cup X^{-\varepsilon}(a, L) \rightarrow X^{-\varepsilon / 2}(a, L)
$$

is an e.h.e. Since $X^{-\varepsilon}(a, L) \rightarrow X^{-\varepsilon / 2}(a, L)$ is an e.h.e., it is enough to prove that

$$
X^{-\varepsilon}(a, L) \rightarrow \tilde{X}^{-\varepsilon / 2}(a, L) \times D_{\infty}\left(K_{0}\right) \cup X^{-\varepsilon}(a, L)
$$

is an e.h.e.

But if $A, B$ are two spaces, in order to prove that $A \rightarrow A \cup B$ is an e.h.e., it is enough to prove that $A \cap B \rightarrow B$ is an e.h.e. Here we set $A=X^{-\varepsilon}(a, L)$, $B=\tilde{X}^{-\varepsilon / 2}(a, L) \times D_{\infty}\left(K_{0}\right)$, and we have to consider the map

$$
X^{-\varepsilon}(a, L) \cap \tilde{X}^{-\varepsilon / 2}(a, L) \times D_{\infty}\left(K_{0}\right) \rightarrow X^{-\varepsilon}(a, L)
$$

induced by the inclusion. In order to prove that this is an e.h.e., we consider the restriction of $F_{L}$ to $\tilde{X}^{-\varepsilon / 2}(a, L) \times D_{\infty}\left(K_{0}\right)$. By the concavity of $F_{L}$ in the direction $D_{\infty}\left(K_{0}\right), F_{L}$ is less than $-\varepsilon / 2$ on this set. By Propositions 1 and 2 of Appendix 1, we just have to prove that $F_{L}$ has no critical point in the interior of $B$ with critical value in $[-\varepsilon,-\varepsilon / 2]$ and that critical points of the restriction of $F_{L}$ to the boundary of $B$ are such that $\nabla F_{L}$ points outward.

Now a critical point $(y, h)$ of $F_{L}$ in the interior of $B$ satisfies

$$
\nabla F_{L}\left(y+z_{L}(y)+h\right)=0
$$

since $F_{L}$ has only zero as a critical point, and $y=0$ is not in $\tilde{X}^{-\varepsilon / 2}(a, L)$, this cannot happen. 
If $(y, h)$ is a critical point of the restriction of $F_{L}$ to the boundary, we have

$$
\nabla F_{L}\left(y+z_{L}(y)+h\right)=\lambda \nabla \widetilde{F}_{L}(y) ;
$$

this implies $\partial F_{L}\left(y+z_{L}(y)+h\right) / \partial h=0$, that is, $h=0$, hence $\lambda=1$, and $\nabla F_{L}\left(y+z_{L}(y)\right)=\nabla \widetilde{F}_{L}(y)$ indeed points outward.

By Proposition 2(i) of Appendix 1, this implies that

$$
B \cap\left\{F_{L} \leq-\varepsilon\right\} \rightarrow B
$$

is an e.h.e.

This concludes our proof, modulo (7.14); that is, we have to prove that $\left\|z_{L}(y)\right\| \leq C\|y\|_{H^{1}}$ with $C$ independent of $L$. This will follow from

$$
\left|\frac{\partial}{\partial L}\left\|z_{L}(y)\right\|\right| \leq\left\|\frac{\partial}{\partial L} z_{L}(y)\right\| \leq C\left(\|y\|_{H^{\prime}}+\left\|z_{L}(y)\right\|\right)
$$

by using Gronwall's lemma.

Let us prove (7.16): for $h \in D_{\infty}\left(K_{0}\right), \quad \nabla F_{L}\left(y+z_{L}(y)\right) \cdot h=0$, so

$$
\begin{aligned}
0 & =\frac{\partial}{\partial L}\left(\nabla F_{L}\left(y+z_{L}(y)\right) \cdot h\right) \\
& =\left(F_{L}^{\prime \prime}\left(y+z_{L}(y)\right) \frac{\partial}{\partial L} z_{L}(y), h\right)+\left(\frac{\partial}{\partial L} \nabla F_{L}\right)\left(y+z_{L}(y)\right) \cdot h .
\end{aligned}
$$

Let us compute $\left(\partial \nabla F_{L} / \partial L\right)(x) \cdot h=\nabla\left(\partial F_{L} / \partial L\right)(x) \cdot h$, using (7.5):

$$
\left(\frac{\partial}{\partial L} \nabla F_{L}\right)(x) \cdot h=-\left\langle x-\nabla H_{L}^{*}(-J \dot{x}+L x), h-H_{L}^{* \prime \prime}(-J \dot{x}+L x)(-J \dot{h}+L h)\right\rangle,
$$

since $h \in D_{\infty}\left(K_{0}\right),-J \dot{h}+L h=\left(L-K_{0}\right) h$, and $\nabla F_{L}\left(y+z_{L}(y)\right) \cdot h=0$ can be written $\left\langle x-\nabla H_{L}^{*}(-J \dot{X}+L x), h\right\rangle=0$, so plugging this into (7.18) yields

$$
\begin{aligned}
& \left(\frac{\partial}{\partial L} \nabla F_{L}\right)\left(y+z_{L}(y)\right) \cdot h \\
& \quad=\left(L-K_{0}\right)\left\langle x-\nabla H_{L}^{*}(-J \dot{x}+L x), H_{L}^{* \prime \prime}(-J \dot{x}+L x) h\right\rangle
\end{aligned}
$$

where $x=y+z_{L}(y)$; as a result,

$$
\left|\left(\frac{\partial}{\partial L} \nabla F_{L}\right)\left(y+z_{L}(y)\right) \cdot h\right| \leq C\left|L-K_{0}\right|\left\|y+z_{L}(y)\right\|_{H^{1}}\|h\| .
$$

The other term in (7.17) is taken care of using the concavity of $F_{L}$ in the direction of $D_{\infty}\left(K_{0}\right)$ (cf. (7.7)) so that

$$
\left|F_{L}^{\prime \prime}\left(y+z_{L}(y)\right)(h, h)\right| \geq C\left|L-K_{0}\right|\|h\|^{2} .
$$

Set $h=\partial z_{L}(y) / \partial L ;$ then (7.17) implies

$$
C\left|L-K_{0}\right|\left\|y+z_{L}(y)\right\|_{H^{\prime}}\left\|\frac{\partial}{\partial L} z_{L}(y)\right\| \geq C\left|L-K_{0}\right|\left\|\frac{\partial}{\partial L} z_{L}(y)\right\|^{2},
$$

hence $\left\|\partial z_{L}(y) / \partial L\right\| \leq C\left(\|y\|_{H^{1}}+\left\|z_{L}(y)\right\|\right)$, which proves (7.16). 
To conclude our proof, we apply Gronwall's lemma; thus

$$
\left\|z_{L}(y)\right\| \leq\left\|z_{L_{0}}(y)\right\| e^{C\left(L-L_{0}\right)}+C\|y\|_{H^{1}}\left(e^{C\left(L-L_{0}\right)}-1\right),
$$

and using the homogeneity of $y \rightarrow z_{L}(y)$, we infer

$$
\left\|z_{L}(y)\right\| \leq C\|y\|_{H^{\prime}} .
$$

This concludes the proof of Lemma 7.9.

We can now prove case (b) of step 1: let $K<K_{0}<K^{\prime}$; then by Lemma 7.9, $\left(X, X^{-}(a, K)\right)$ has the e.h. type of

$$
\left(C\left(K_{0}\right) \times D\left(K_{0}\right), \tilde{X}^{-}\left(a, K_{0}\right) \times D\left(K_{0}\right)\right),
$$

and $\left(X, X^{-}\left(a, K^{\prime}\right)\right)$ has the e.h. type of

$$
\left(C\left(K_{0}\right) \times D_{\infty}\left(K_{0}\right), \tilde{X}^{-}\left(a, K_{0}\right) \times D_{\infty}\left(K_{0}\right) \cup \complement \overline{C\left(K_{0}\right) \times D\left(K_{0}\right)}\right) .
$$

We denote these pairs respectively by

$$
\left(C\left(K_{0}\right), \tilde{X}^{-}\left(a, K_{0}\right)\right) \times D\left(K_{0}\right)
$$

and

$$
\left(C\left(K_{0}\right), \tilde{X}^{-}\left(a, K_{0}\right)\right) \times\left(D_{\infty}\left(K_{0}\right), C \overline{D\left(K_{0}\right)}\right) .
$$

Now let $\gamma:\left(\Gamma_{1}, \Gamma_{2}\right) \rightarrow\left(X, X^{-}(a, K)\right)$ represent some homology class as described in the sketch of step 1 , and consider the commutative diagram in Figure 1.

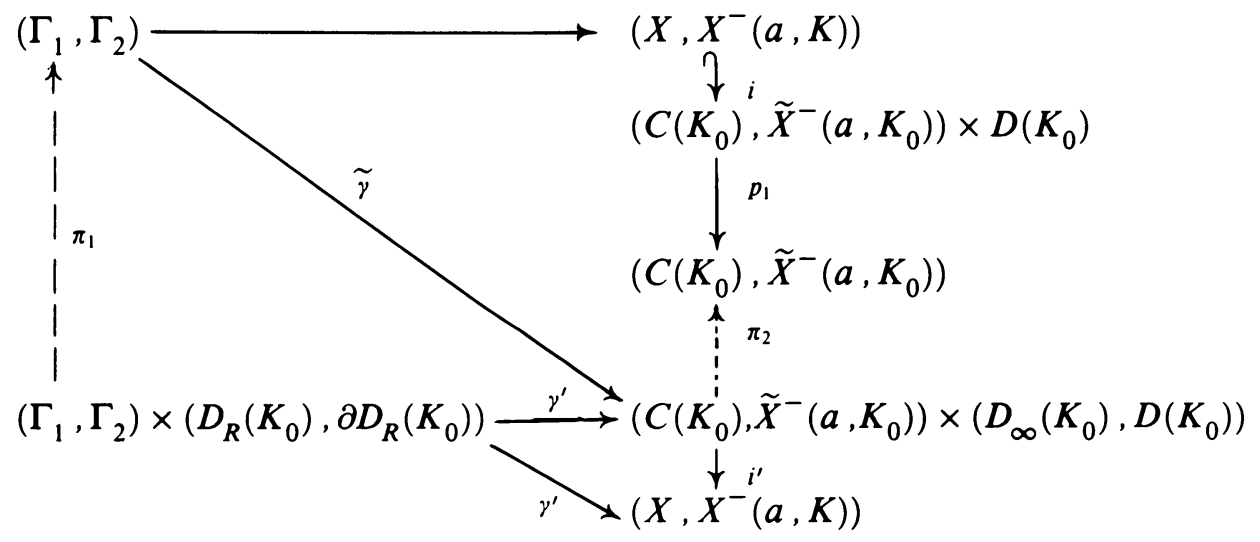

FIGURE 1

In the diagram, $i$ and $i^{\prime}$ are the maps (7.10) and (7.11) of Lemma 7.9, $p_{1}$ the projection on the first factor. The dashed arrows $\pi_{1}, \pi_{2}$ represent the fibration $\Gamma_{1} \times D_{R}\left(K_{0}\right) \rightarrow \Gamma_{1}$ and the fibration $C\left(K_{0}\right) \times D_{\infty}\left(K_{0}\right) \rightarrow C\left(K_{0}\right)$. The map $\tilde{\gamma}^{\prime}$ is defined by

$$
\tilde{\gamma}^{\prime}(x, u)=\left(p_{1} i \gamma(x), u\right) .
$$

Let us check that $\tilde{\gamma}^{\prime}\left(\Gamma_{1} \times \partial D_{R}\left(K_{0}\right) \cup \Gamma_{2} \times D_{R}\left(K_{0}\right)\right)$ is contained in $\overline{C C\left(K_{0}\right) \times D\left(K_{0}\right)}$ $\cup \tilde{X}^{-}\left(a, K_{0}\right) \times D_{\infty}\left(K_{0}\right)$ for $R$ large enough. 
To begin with, $\tilde{\gamma}^{\prime}$ embeds $\Gamma_{2} \times D_{R}\left(K_{0}\right)$ into $\tilde{X}^{-}\left(a, K_{0}\right) \times D_{\infty}\left(K_{0}\right)$, since by assumption, $\gamma\left(\Gamma_{2}\right)$ is contained in $X^{-}(a, K) \subset \widetilde{X}^{-}\left(a, K_{0}\right) \times D_{\infty}\left(K_{0}\right)$, thus implying

$$
\tilde{\gamma}^{\prime}\left(\Gamma_{2} \times D_{R}\left(K_{0}\right)\right) \subset p_{1} i \gamma\left(\Gamma_{2}\right) \times D_{\infty}\left(K_{0}\right) \subset \tilde{X}^{-}\left(a, K_{0}\right) \times D_{\infty}\left(K_{0}\right) .
$$

We now prove that $\tilde{\gamma}^{\prime}\left(\Gamma_{1} \times \partial D_{R}\left(K_{0}\right)\right)$ is contained in $\complement \overline{C\left(K_{0}\right) \times D\left(K_{0}\right)}$. This is obvious, since $\Gamma_{1}$ being compact, for $R$ large enough $p_{1} i \gamma\left(\Gamma_{1}\right) \times \partial D_{R}\left(K_{0}\right)$ is contained in $\complement \overline{C\left(K_{0}\right) \times D\left(K_{0}\right)}$ (just take $\left.R \geq \sup \left\{\phi(y) \mid y \in p_{1} i \gamma\left(\Gamma_{1}\right)\right\}\right)$.

Now, according to Appendix 3, Proposition 2, corresponding to the Thom isomorphisms of $\pi_{1}$ and $\pi_{2}$, we have two natural isomorphisms,

$$
\begin{aligned}
T_{1} & : H_{S^{1}, *}\left(\Gamma_{1}, \Gamma_{2}\right) \rightarrow H_{S^{1}, *+2 n}\left(\left(\Gamma_{1}, \Gamma_{2}\right) \times\left(D_{R}\left(K_{0}\right), \partial D_{R}\left(K_{0}\right)\right)\right), \\
T_{2} & : H_{S^{1}, *}\left(C\left(K_{0}\right), \tilde{X}^{-}\left(a, K_{0}\right)\right) \\
& \rightarrow H_{S^{1}, *+2 n}\left(\left(C\left(K_{0}\right), \tilde{X}^{-}\left(a, K_{0}\right)\right) \times\left(D_{\infty}\left(K_{0}\right), \underline{C} \underline{D} D\left(K_{0}\right)\right)\right) .
\end{aligned}
$$

Since $\tilde{\gamma}^{\prime}$ is a morphism between the fibrations $\pi_{1}, \pi_{2}$ which induces $\tilde{\gamma}$ on the base, we have by naturality

$$
\text { - } \tilde{\gamma}_{*}^{\prime} \circ T_{1}=T_{2} \circ \tilde{\gamma}_{*} .
$$

Hence, by using the diagram, this yields

$$
\gamma_{*}^{\prime} \circ T_{1}=i_{*}^{\prime} \circ T_{2} \circ p_{1, *} \circ i_{*} \circ \gamma_{*} .
$$

Since $i_{*}^{\prime}$ and $i_{*}$ are, according to Lemma 6.9, isomorphisms, $p_{1, *}$ and $T_{2}$ are isomorphisms as well, and the map (7.2) which is equal to $i_{*}^{\prime} \circ T_{2} \circ p_{1, *} \circ i_{*}$ is an isomorphism.

Thus as announced in the sketch of step 1, there is an isomorphism

$$
\lambda_{*}: H_{S^{1}, *}\left(X, X^{-}(a, K)\right) \rightarrow H_{S^{1}, *+2 n}\left(X, X^{-}\left(a, K^{\prime}\right)\right)
$$

given geometrically by $\gamma \rightarrow \gamma^{\prime}=\gamma \oplus D_{R}\left(K_{0}\right)$, or to be more precise such that $\gamma_{*}^{\prime} \circ T_{1}=\lambda_{*} \circ \gamma_{*}$.

This concludes the proof of case (b) of Step 1, and thus the proof of Step 1 is complete.

Step 2. We prove that for given $a, K$, and for $a^{\prime}, K^{\prime}$ large enough, the map

$$
T: H_{S^{1}, k+d(K)}\left(X, X^{-}(a, K)\right) \rightarrow H_{S^{1}, k+d\left(K^{\prime}\right)}\left(X, X^{-}\left(a^{\prime}, K^{\prime}\right)\right)
$$

is zero. This map is obtained by comparing the map induced by the embedding $X^{-}(a, K) \hookrightarrow X^{-}\left(a^{\prime}, K^{\prime}\right)$ and the map from $H_{S^{1}, k+d(K)}\left(X, X^{-}(a, K)\right)$ into $H_{S^{1}, k+d\left(K^{\prime}\right)}\left(X, X^{-}\left(a, K^{\prime}\right)\right)$ obtained in Step 1.

We shall show that for any compact pair $\left(\Gamma_{1}, \Gamma_{2}\right)$ and map $\gamma:\left(\Gamma_{1}, \Gamma_{2}\right) \rightarrow$ $\left(X, X^{-}(a, K)\right)$,

$$
T \circ \gamma_{k}=0 \text { for } k \in \stackrel{\circ}{I} \text {. }
$$


To begin with, we use the finite-dimensional reduction of $\S 3$, in order to deform $\gamma$ into a map whose image is in $E_{N}=\bigoplus_{k=-N}^{N} D_{\infty}(k)$, by applying Lemma 5.1. We still denote this map by $\gamma$.

Using step $1, T \circ \gamma_{*}$ is represented geometrically by $\gamma^{\prime}=\gamma \oplus D_{R}\left(K, K^{\prime}\right)$, whose image is then contained in $E_{N} \oplus \bigoplus_{K<k<K^{\prime}} D_{\infty}(k)$.

On the other hand, we have

$$
H_{K}(x) \leq \frac{1}{2}\left(K+a / R^{2}\right)|x|^{2}
$$

hence

which implies that

$$
H_{K}^{*}(y) \leq \frac{R^{2}}{2\left(K R^{2}+a\right)}|y|^{2},
$$

$$
F_{a, K}(x) \leq \frac{R^{2}}{2\left(K R^{2}+a\right)} \int_{0}^{T}\left(J \dot{x}-K x, J \dot{x}+\frac{a}{R^{2}} x\right) d s .
$$

Writing down the Fourier series of $x, x=\sum_{k \in \mathbf{Z}} \exp ((2 \pi / T) k J t) x_{k}$, the latter inequality yields

$$
F_{a, K}(x) \leq \frac{R^{2}}{2\left(K R^{2}+a\right)} \sum_{k \in \mathbf{Z}}\left(\frac{2 \pi k}{T}+K\right)\left(\frac{2 \pi k}{T}+\frac{a}{R^{2}}\right)\left|x_{k}\right|^{2} .
$$

Finally, for $x \in \bigoplus_{-K<k \leq k(a)} D_{\infty}(k)$, where $k(a)$ is defined to be $a / R^{2}$, $F_{a, K}(x)<0$, i.e., $x \in X^{-}(a, K)$. It is now clear that for $a^{\prime}, K^{\prime}$ large enough,

$$
E_{N} \oplus \bigoplus_{-K^{\prime}<k<-K} D_{\infty}(k)=\bigoplus_{-K^{\prime}<k \leq N} D_{\infty}(k)
$$

is contained in $X^{-}\left(a^{\prime}, K^{\prime}\right)$, hence the image of $\gamma^{\prime}$ is contained in $X^{-}\left(a^{\prime}, K^{\prime}\right)$, thus $\gamma_{*}^{\prime}$ is zero.

This concludes the proof of Step 2.

Step 3. We assume $\mathbf{F}=\mathbf{Q}$. We wish to prove that the map

$$
H_{S^{1}, k+d(K)}\left(X, X^{-}(a, K)\right) \rightarrow H_{S^{1}, k+d\left(K^{\prime}\right)}\left(X, X^{-}\left(a^{\prime}, K^{\prime}\right)\right) \text {, }
$$

induced by the embedding $X^{-}\left(a, K^{\prime}\right) \rightarrow X^{-}\left(a^{\prime}, K^{\prime}\right)$, is an isomorphism for $k \in \stackrel{\circ}{I}$. This is equivalent to proving that

$$
H_{S^{1}, k+d\left(K^{\prime}\right)}\left(X^{-}\left(a^{\prime}, K^{\prime}\right), X^{-}(a, K)\right)=0 \text { for } k \in \stackrel{\circ}{I},
$$

as we easily see by using the cohomology exact sequences of the pairs

$$
\left(X, X^{-}\left(a, K^{\prime}\right)\right), \quad\left(X, X^{-}\left(a^{\prime}, K^{\prime}\right)\right), \quad\left(X^{-}\left(a^{\prime}, K^{\prime}\right), X^{-}(a, K)\right)
$$

and the five lemma.

By homogeneity, (7.24) is equivalent to

$$
H_{S^{1}, k+d\left(K^{\prime}\right)}\left(X^{-}\left(a^{\prime}, K^{\prime}\right) \cap S, X^{-}\left(a, K^{\prime}\right) \cap S\right)=0 \quad \text { for } k \in \stackrel{\circ}{I} \text {, }
$$

where $S$ is the unit sphere. 
To check (7.25), we apply the corollary of Proposition 3 of Appendix 1, taking for $f_{t}$ the restriction of $F_{K^{\prime}, t}$ to $S$.

We already checked for Step 1 that the critical points of $F_{K^{\prime}, t}$ correspond to $t \cdot T / 2$-periodic solutions of $(\mathscr{N})$. By assumption, their index is $i+d\left(K^{\prime}\right)$ with $i$ not in $I$. Since $\mathbf{F}=\mathbf{Q}, H_{q}\left(B G_{x}, \theta\right)=0$ for $g \neq 0$, where $G_{x}$ is the isotropy group of the orbit, we can apply the above-mentioned corollary and conclude that $(7.25)$ holds for $k \in \stackrel{\circ}{I}$.

This completes the proof of Theorem 7.1.

\section{Proving Theorem 1.2}

We shall now write the equivariant Morse inequalities for $F_{a, K}$ in order to prove Theorem 1.2.

As noted at the beginning of $\S 7$, the origin is the only critical orbit in $X-X^{-\varepsilon}$. Hence in the exact homology sequence of the triple $\left(X, X^{-\varepsilon}, X^{-b}\right)$ (for $b$ large as in $\S 6)$,

$$
\begin{aligned}
\rightarrow H_{S^{1}, *}\left(X^{-\varepsilon}, X^{-b}\right) & \rightarrow H_{S^{1}, *}\left(X, X^{-b}\right) \\
& \rightarrow H_{S^{1}, *}\left(X^{-\varepsilon}, X^{-b}\right) \rightarrow H_{S^{1}, *-1}\left(X^{-\varepsilon}, X^{-b}\right) \rightarrow \cdots
\end{aligned}
$$

the term $H_{S^{1}, *}\left(X^{-\varepsilon}, X^{-h}\right)$ corresponds to the contribution of the periodic orbits of $(\mathscr{N})$ of period $\leq a T / 2$. We shall denote by $M_{a}(t)=\sum_{k \in \mathbf{Z}} m_{k}(a) t^{k}$ the normalized Morse series of $F_{a, K}$ in $X^{-\varepsilon}-X^{-b}$. If we denote by $t^{d(K)} H_{a}(t)$ the Poincaré series of $H_{S^{1}, *}\left(X^{-\varepsilon}, X^{-b}\right), H_{a}(t)$ is a Laurent series, and we have the equivariant Morse inequality

$$
M_{a}(t)-H_{a}(t)=(1+t) R_{a}(t),
$$

where $R_{a}(t)$ is a Laurent series with nonnegative coefficients (cf. Appendix 1).

On the other hand, the Poincare series of $H_{S^{1}, *}\left(X, X^{-b}\right)$ is, by Corollary 5.11, $t^{d(K)}\left(1 /\left(1-t^{2}\right)\right)$. The Poincaré series of $H_{S^{1}, *}\left(X, X^{-\varepsilon}\right)$ is $t^{d(K)} Q_{a}(t)$, where according to Theorem 7.1 , if we set $Q_{a}(t)=\sum_{k \in \mathbf{Z}} q_{k}(a) t^{k}$ then

$$
q_{k}(a)=0 \text { for } k \in \stackrel{\circ}{I}
$$

(we suppose $\mathbf{F}=\mathbf{Q}$ and $\stackrel{\circ}{I}$ is defined as in Theorem 7.1).

Now from (8.1) we have

$$
H_{a}(t)-\frac{1}{1-t^{2}}+Q_{a}(t)=(1+t) S_{a}(t)
$$

with $S_{a}(t)$ a Laurent series with nonnegative coefficients. Adding up (8.2) and (8.4) yields

$$
M_{a}(t)-\frac{1}{\left(1-t^{2}\right)}+Q_{a}(t)=(1+t) U_{a}(t)
$$

where $U_{a}$ also has nonnegative coefficients. 
Now truncate (8.4) at the degrees $2 C$ and $2 N$, where we set $C$ equal to $2 n^{2}$, and $2 N>2 C$, and write $M_{a}^{2 N}(2 C ; t), Q_{a}^{2 N}(2 C ; t) \cdots$ for the truncated series; then from (8.4) we infer

$$
\begin{aligned}
& M_{a}^{2 N}(2 C ; t)-\sum_{h=C}^{N} t^{2 h}+Q_{a}^{2 N}(2 C ; t) \\
& \quad=(1+t) U_{a}^{2 N-1}(2 C ; t)+t^{2 N} u_{2 N}(a)+t^{2 C} u_{2 C-1}(a) .
\end{aligned}
$$

By (8.3), and the fact that for $a$ large enough $\stackrel{\circ}{I}$ contains $[2 C, 2 N]$, indeed let $\alpha$ such that any solution with $\hat{\imath} \neq 0$ has $|\hat{\imath}|>\alpha$ and period smaller than $\tau$. Then if $k \geq a T / 2, i_{k} \sim k \hat{\imath} \geq k a \geq(a T / 2) \alpha$, which goes to infinity with $a$ so $Q_{a}^{2 N}(2 C ; t)=0$, and $(8.6)$ can be written

$$
M_{a}^{2 N}(2 C ; t)-\sum_{h=C}^{N} t^{2 h}=(1+t) U_{a}^{2 N-1}(2 C ; t)+t^{2 N} u_{2 N}(a)+t^{2 C} u_{2 C-1}(a)
$$

So, if in (8.7) we let first $a$, then $N$, go to infinity, we get

$$
M^{+}(2 C ; t)-\sum_{h=C}^{N} t^{2 h}=(1+t) U^{+}(2 c ; t)+t^{2 C} u_{2 C-1},
$$

where $u_{2 C-1} \geq 0$ and $U(2 c ; t)$ has nonnegative coefficients.

Changing $C$ into $-C, N$ into $-N$, we obtain

$$
M^{-}(2 C ; t)=(1+t) U^{-}(2 C ; t)+t^{-2 C} u_{-2 C} .
$$

Let us now check that all series have bounded coefficients. In fact, it is enough to check that $M^{+}(2 C ; t)$ has bounded coefficients. Now $m_{l}$ is bounded by the number of solutions of index $l$. Consider a primitive solution with nonnegative $\hat{\imath}$; then by assertion (i) of Proposition 6.14 , we have $i_{k} \in\left[k \hat{\imath}-c, k \hat{\imath}+c^{\prime}\right]$ so $i_{k}=l$ for a most $\left(c+c^{\prime}\right) / \hat{\imath}$ values of $k$, whence

$$
m_{l} \leq \sum \frac{c+c^{\prime}}{\hat{l}}
$$

where the sum is taken on the set primitive solutions with nonnegative $\hat{\imath}$, thus proving the boundedness of $m_{l}$.

Now multiply $(8.8)$ by $(1+t)$ and take the limit as $t$ goes to -1 . We get by Proposition 6.18

$$
\sum^{\prime} \frac{(-1)^{i_{1}}}{\hat{\imath}}+\sum^{\prime \prime} \frac{(-1)^{i_{1}}}{2 \hat{\imath}}=\frac{1}{2}
$$

where $\sum^{\prime}$ and $\sum^{\prime \prime}$ have the same meaning as in Theorem 1.2.

Doing the same with (8.9) yields

$$
\sum^{\prime \prime \prime} \frac{(-1)^{i_{1}}}{\hat{\imath}}+\sum^{(\mathrm{iv})} \frac{(-1)^{i_{1}}}{2 \hat{\imath}}=0
$$


Let us also mention that if there is no solution with $\hat{\imath}=0$ we do not need to cut our series at $\pm 2 C$; we can cut at $-2 N$ and $2 N$, thus obtaining

$$
M(t)-\frac{1}{1-t^{2}}=(1+t) U(t) .
$$

\section{THE CASE OF FINITELY MANY HYPERBOLIC ORBITS}

Suppose now that $(\mathcal{N})$ has only a finite number of primitive hyperbolic solutions. We shall prove that this cannot happen if $n$ is even.

Let us first remark that for a hyperbolic solution $i_{k}=(k-1) \hat{\imath}+i_{1}$ and $\hat{\imath}=j(-1)=i_{1}+n+1$. So if $\hat{\imath}>0$ the contribution of such a solution and its iterates is

$$
\begin{aligned}
& t^{-(m-1)} \frac{t^{\hat{i}}}{1-t^{\hat{i}}} \text { for } \hat{\imath} \text { even }, \\
& t^{-(m-1)} \frac{t^{\hat{i}}}{1-t^{2 \hat{\imath}}} \text { for } \hat{\imath} \text { odd } .
\end{aligned}
$$

There are no orbits with $\hat{\imath}=0$, because such an orbit and all its iterates would have the same index, $-(n+1)$. Now for $a$ large enough all the orbits of period larger than $a T / 2$ will have their index

$$
\begin{array}{ll}
\text { either (i) equal to }-(n+1) \\
\text { or } & \text { (ii) different from }-(n+1) \pm 1 \text {. }
\end{array}
$$

This implies that for large values of $a, a^{\prime}, a<a^{\prime}$,

$$
H_{S^{1}, k+d(K)}\left(X, X^{-}(a, K)\right) \rightarrow H_{S^{\prime}, k+d(K)}\left(X, X^{-}\left(a^{\prime}, K\right)\right),
$$

induced by the inclusion map $X^{-}\left(a^{\prime}, K\right) \hookrightarrow X^{-}(a, K)$, is nonzero for $k=$ $-(n+1)$, which contradicts step 2 of $\S 7$. To prove it, we first claim that (9.3) is injective: "going" from $X^{-}(a, K)$ to $X^{-}\left(a^{\prime}, K\right)$ we "meet" periodic orbits of type (i) or (ii) described above. None of these can "kill" a homology class of dimension $d(K)-(n+1)$ so $(9.3)$ is injective. On the other hand, $H_{S^{1}, d(K)-(n+1)}\left(X, X^{-}(a, K)\right)$ is nonzero since when $a$ increases, we "meet" infinitely many orbits of type (i). Such an orbit can either contribute to $H_{S^{1} . d(K)-(n+1)}\left(X, X^{-}(a, K)\right)$ or kill a homology class of dimension $d(K)-$ $n-2$. But the latter are finite, because only finitely many orbits have index $d(K)-n-2$; so infinitely many orbits of type (i) must actually contribute to $H_{S^{1}, d(K)-(n+1)}\left(X, X^{-}(a, K)\right)$, which is then nonzero. This concludes the proof.

As there are no orbits of index $-(n+1)$, we can split the Morse inequalities in two, one corresponding to orbits of index $>-(n+1)$, the other to the orbits of index $<-(n+1)$, which amounts to separating the orbits with $\hat{\imath}>0$ from those with $\hat{\imath}<0$.

In the sequel we consider only the Morse inequalities obtained for $\hat{\imath}>0$, that is,

$$
t^{-(n+1)}\left(\sum_{l \text { even }} \frac{t^{i}}{1-t^{i}}+\sum_{i \text { odd }} \frac{t^{i}}{1-t^{2 i}}\right)-\frac{1}{1-t^{2}}-(1+t) R(t),
$$

and $R$ has nonnegative coefficients. 
Now write $c_{k}$ for the number of orbits with $\hat{\imath}=k$; then (9.4) is equivalent to

$$
\begin{aligned}
\sum_{h \geq 1} c_{2 h} & {\left[\frac{N}{2 h}\right]-\sum_{h \geq 0} c_{2 h+1}\left[\frac{N}{2(2 h+1)}+\frac{1}{2}\right] } \\
& -(-1)^{n+1} \sup \left(\left[\frac{N-(n+1)}{2}+1\right] ; 0\right)=(-1)^{N} r_{N},
\end{aligned}
$$

where $t^{(n+1)} R(t)=\sum r_{k} t^{k}$.

Now obviously, by looking at the leading term (or applying Theorem 1.2),

$$
\sum \frac{c_{2 h}}{2 h}-\sum \frac{c_{2 h+1}}{2(2 h+1)}=0
$$

Now let $q$ be an integer such that $c_{l}=0$ for $l \geq q$, and apply (9.5) to $N=q !-2$. Then $[(q !-2) / 2 h]=(q ! / 2 h)-1$ if $c_{2 h} \neq 0$, and

$$
\begin{aligned}
{\left[\frac{q !-2}{2(2 h+1)}+\frac{1}{2}\right] } & =\frac{q !}{2(2 h+1)}+\left[\frac{1}{2}-\frac{1}{2 h+1}\right] \\
& =\frac{q !}{2(2 h+1)} \quad \text { if } h \neq 0, \\
& =\frac{q !}{2(2 h+1)}-1 \quad \text { if } h=0 .
\end{aligned}
$$

So (9.5) can be written

$$
\begin{aligned}
\sum_{h \geq 1} c_{2 h}\left(\frac{q !}{2 h}-1\right) & -\sum_{h \geq 1} c_{2 h+1} \frac{q !}{2(2 h+1)} \\
& -c_{1}\left(\frac{q !}{2}-1\right)-(-1)^{n+1}\left[\frac{q !}{2}-\frac{n+1}{2}\right]=r_{N} \geq 0 ;
\end{aligned}
$$

using (2.6) this becomes, for $n$ even,

$$
-\sum_{h \geq 1} c_{2 h}+c_{1}-\frac{n}{2} \geq 0
$$

Applying (9.5) with $N=2$ yields

$$
c_{2}-c_{1} \geq 0
$$

adding (9.8) and (9.9) gives

$$
-\sum_{h \geq 2} c_{2 h}-\frac{n}{2} \geq 0
$$

which is rather unlikely to happen.

\section{APPENDIX 1: MORSE THEORY WITH BOUNDARY AND SOME COROLLARIES}

Let $X$ be a smooth manifold with boundary, and $f$ a $C^{2}$ function satisfying condition (C) defined on $X$. We set $X^{a}=\{x \in X \mid f(x) \leq a\}$; then the following holds. 
Proposition 1. (i) If $f$ and $\left.f\right|_{\partial X}$ have no critical values in $[a, b]$, then $X^{a} \approx$ $X^{b}$.

(ii) If $\left.f\right|_{\partial X}$ has no critical value in $[a, b]$ and $f$ has a single nondegenerate critical point in $f^{-1}([a, b])$ of index $i$, then

$$
H_{k}\left(X^{b}, X^{a}\right)= \begin{cases}\mathbf{F} & \text { if } k=i \\ 0 & \text { otherwise } .\end{cases}
$$

The same is true in the equivariant setting, that is, if $f$ is G-invariant, $H_{*}$ replaced by $H_{G, *}$, and the last two equalities replaced by

$$
H_{G ; k}\left(X^{b}, X^{a}\right) \simeq H_{k-i}\left(B G_{x}, \theta\right),
$$

where $G_{x}$ is the isotropy group of the critical orbit, $\theta$ the orientation bundle of the negative bundle of $f^{\prime \prime}(x)$.

Proof. This is standard Morse theory.

Proposition 2. If in $f^{-1}[a, b]$ there is a single nondegenerate critical point, $x_{0}$, of $\left.f\right|_{\partial X}$ of index $i$, then

(i) if $\nabla f\left(x_{0}\right)$ points outward, $X^{b} \approx X^{a}$;

(ii) if $\nabla f\left(x_{0}\right)$ points inward, then $X^{b}$ is diffeomorphic to $X^{a}$ with an $i$ dimensional handle attached.

Also if $\{x \in X \mid f(x)=a\}$ is denoted by $X(a)$ we have, in case

(i) $X(a)$ is diffeomorphic to $X(b)$ with an i-dimensional handle attached;

(ii) $X(b)$ is diffeomorphic to $X(a)$ with an i-dimensional handle attached.

The same is true in the equivariant setting.

Corollary. With the hypothesis of Proposition 2, we have

$$
\begin{aligned}
& H_{k}\left(X^{b}\right) \approx H_{k}\left(X^{a}\right) \quad \text { for } k \neq i, i-1, \\
& H_{k}(X(b)) \approx H_{k}(X(a)) \text { for } k \neq i, i-1 \text {. }
\end{aligned}
$$

In the equivariant setting, this is true provided the condition $k \neq i, i-1$ is replaced by

$$
H_{k-i}\left(B G_{x_{0}}, \theta\right)=0, \quad H_{k-i+1}\left(B G_{x_{0}}, \theta\right)=0
$$

(cf. Proposition 1).

Proof. This is just Morse theory with boundary.

Consider now $M$ a manifold without boundary, $f_{t}$ a family of functions on $M$ satisfying condition (C). Set $f(t, x)=f_{t}(x)$, and assume $f$ is $C^{2}$. Denote $\left\{x \in M \mid f_{t}(x) \leq a\right\}$ by $M_{t}^{a}$; then we have

Proposition 3. (a) If $f_{t}$ has no critical point of level 0 for $t \in[a, b]$, then $M_{a}^{0} \approx M_{b}^{0}$. 
(b) If $f_{0}$ has a unique nondegenerate critical point, $x_{0}$, of index $i$ in $f^{-1}(0)$, then

(i) if $\partial f_{t}\left(x_{0}\right) / \partial t<0$, then $M^{b}$ is diffeomorphic to $M^{a}$ with an $i$ dimensional bundle attached;

(ii) if $\partial f_{t}\left(x_{0}\right) / \partial t>0$, then $M^{a}$ is diffeomorphic to $M^{b}$ with an $i$ dimensional handle attached.

The same is true in an equivariant setting.

Proof. Set $X=\left\{(x, t) \in M \times \mathbf{R} \mid f_{t}(x) \leq 0\right\}$; this is a manifold with boundary as $d f(x, t)=d_{x} f_{t}(x) d x+(\partial / \partial t) f_{t}(x) d t$ is never zero.

Consider on $X$ the map $\tau:(x, t) \rightarrow t$. This map has no critical points in the interior of $X .\left(x_{0}, t_{0}\right)$ is a critical point of $\left.t\right|_{\partial x}$ if and only if $d t=\lambda d f\left(x_{0}, t_{0}\right)$, that is, $d_{x} f_{t_{0}}\left(x_{0}\right)=0$ and $\partial f_{t_{0}}\left(x_{0}\right) / \partial t \neq 0$, and $\nabla \tau$ points inward if and only if $\lambda<0$, that is, $\partial f_{t}\left(x_{0}\right) / \partial t<0$.

Applying Proposition 2 yields the result.

Corollary. $H_{k}\left(M_{a}^{0}\right) \approx H_{k}\left(M_{b}^{0}\right)$ for $k \neq i, i-1$, and the same is true in the equivariant setting with the condition $k \neq i, i-1$ changed as in the corollary of Proposition 2.

Proof. Apply the corollary of Proposition 2.

\section{APPENDIX 2: MORSE INEQUALITIES}

In this appendix, $A_{k}, B_{k}, \ldots$ will denote graded vector spaces and $A(t)$, $B(t)$ the Poincare series, that is,

$$
A(t)=\sum_{k \in \mathbf{Z}} t^{k} \operatorname{dim} A_{k} .
$$

Suppose now that we have a long exact sequence

$$
A_{*} \rightarrow B_{*} \rightarrow C_{*} \rightarrow A_{*-1} \rightarrow \cdots \text {; }
$$

then

Proposition 1. $A(t)-B(t)+C(t)=(1+t) R(t)$, where $R(t)$ has nonnegative coefficients.

Proof. Suppose first we have a short exact sequence $0 \rightarrow A_{*} \rightarrow B_{*} \rightarrow C_{*} \rightarrow$ $D_{*} \rightarrow E_{*} \rightarrow 0$; then if we set $a_{k}=\operatorname{dim} A_{k}, b_{k}=\operatorname{dim} B_{k}, \ldots$, we have

$$
a_{k}-b_{k}+c_{k}-d_{k}+e_{k}=0,
$$

hence $A(t)-B(t)+C(t)-D(t)+E(t)=0$. Now from (1) there is a short exact sequence

$$
0 \rightarrow \operatorname{Im} C_{*+1} \rightarrow A_{*} \rightarrow B_{*} \rightarrow C_{*} \rightarrow \operatorname{Im} C_{*} \rightarrow 0,
$$

where $\operatorname{Im} C_{*}$ is the image of $C_{*}$ in $A_{*-1}$. So if $C^{\prime}(t)$ is the Poincare series of $\operatorname{Im} C_{*+1}$, we get

$$
C^{\prime}(t)-A(t)+B(t)-C(t)+t C^{\prime}(t)=0,
$$

that is, $A(t)-B(t)+C(t)=(1+t) C^{\prime}(t)$. 


\section{APPENDIX 3: THOM ISOMORPHISM AND EQUIVARIANT HOMOLOGY}

Let $G$ be a compact group; then up to a homotopy equivalence, there is a unique contractible space $E G$, endowed with a free $G$-action.

Definition 1. $B G=E G / G$ is the classifying space of $G$. The principal $G$ bundle $E G \rightarrow B G$ is the universal $G$-bundle.

Definition 2. If $X$ is a $G$-space, the homotopy quotient of $X$ is $X_{G}=X \times E G / G$ where $G$ operates diagonally on $X \times E G$ (i.e., $g(x, e)=(g x, g e))$.

Remark. If the action of $G$ is free, then $X_{G} \simeq(X / G) \times E G$ has the homotopy type of $X / G$.

Now let $H_{*}$ be some homology theory; we set

Definition 3. If $X$ is a $G$-space, $H_{G, *}(X)=H_{*}\left(X_{G}\right)$.

Now let $\pi: X \rightarrow A$ be a $k$-dimensional $G$-vector bundle, that is, a vector bundle such that $X$ is endowed with a fiber-preserving $G$-action, linear on the fibers.

Let $D(X)$ be the unit disk bundle associated with $X$ (we can choose a $G$ invariant metric, so that $D(X)$ is a $G$ space). Then

Proposition 1. There is a natural isomorphism

$$
T: H_{G, *}(X, X-D(X)) \approx H_{G, *-k}(A ; \theta),
$$

where $\theta$ is the orientation bundle of $\pi$.

Proof. This follows from the fact that we have a vector bundle $X_{G} \rightarrow A_{G}$, and the usual Thom isomorphism.

If we have $K \subset A$ and $Y=\pi^{-1}(K)$, Proposition 1 can be generalized to

Proposition 2. There is a natural isomorphism

$$
T: H_{G, *}(X, Y \cup X-D(x)) \approx H_{G, *-k}(A, K ; \theta) .
$$

\section{REFERENCES}

[A-Z] H. Amann and E. Zehnder, Periodic solutions of asymptotically linear Hamiltonian systems, Manuscripta Math. 32 (1980), 149-189.

[BLMR] H. Berestycki, J. M. Lasry, G. Mancini and B. Ruf, Existence of multiple periodic orbits on starshaped Hamiltonian systems, Comm. Pure Appl. Math. 38 (1985), 253-289.

[Bo1] A. Borel, Cohomologie des espaces localement compacts d'après Leray, Lecture Notes in Math., no. 2, Springer-Verlag, New York, 1984.

[Bo2] _ Seminar on transformation groups, Ann. of Math. Studies, no. 46, Princeton Univ. Press, Princeton, N.J., 1960.

[B1] R. Bott, Lectures on Morse theory, old and new, Bull. Amer. Math. Soc. (N.S.) 7 (1982), 331-358.

[B2] $\_$On the iteration of closed geodesics and the Sturm intersection theory, Comm. Pure Appl. Math. 9 (1956), 176-206. 
[Br] V. Brousseau, Thèse de $3^{\text {ème }}$ cycle, Université de Paris IX, 1985.

[C] F. Clarke, Periodic solutions to Hamiltonian inclusions, J. Differential Equations 40 (1980), 1-6.

[C-E] F. Clarke and I. Ekeland, Hamiltonian trajectories having prescribed minimal period, Comm. Pure Appl. Math. 37 (1980), 103-116.

[Ca] A. Cambini, Sul lemma di Morse, Boll. Un. Mat. Ital. 7 (1973), 87-93.

[Co] C. C. Conley, Isolated invariant sets and their Morse index, CBMS Regional Conf. Ser. in Math., no. 38, Amer. Math. Soc., Providence, R.I., 1978.

[C-Z] C. Conley and E. Zehnder, Morse type index theory for flows and periodic solutions for Hamiltonian equations, Comm. Pure Appl. Math. 37 (1984), 207-253.

[E1] I. Ekeland, Une théorie de Morse pour les systèmes Hamiltoniens convexes, Ann. Inst. H. Poincaré: Anal. Non Linéaire 1 (1984), 19-78.

[E2] _ Index theory for periodic solutions of convex Hamiltonian systems, Cahiers de M. D. no. 85003, Université de Paris IX, 1985.

[E-L] I. Ekeland and J. M. Lasry, On the number of periodic trajectories for a Hamiltonian flow on a convex energy surface, Ann. of Math. (2) 112 (1980), 283-319.

[F-H-R] E. R. Fadell, S. Y. Husseini and P. H. Rabinowitz, Borsuk-Ulam theorems for arbitrary $S^{1}$ actions and applications, Trans. Amer. Math. Soc. 274 (1982), 345-360.

[R1] P. H. Rabinowitz, Periodic solutions of Hamiltonian systems, Comm. Pure Appl. Math. 31 (1978), 157-184.

[Sp] E. Spanier, Algebraic topology, 2nd ed., Springer-Verlag, New York, 1975.

[St] N. Steenrod, The topology of fibre bundles, Princeton Univ. Press, Princeton, N.J., 1951.

[Ta] F. Takens, Hamiltonian systems, generic properties of closed orbits and local perturbations, Math. Ann. 188 (1970), 304-312.

[V1] C. Viterbo, Thèse de $3^{\text {ème }}$ cycle, Université de Paris IX, 1985.

[V2] __ Une théorie de Morse pour les systèmes hamiltoniens étoilés, C. R. Acad. Sci. Paris Ser. I Math. 301 (1985), 487-489.

[V3] __, Intersections de sous-variétés lagrangiennes, fonctionelles d'action et indice des systèmes hamiltoniens, Bull. Soc. Math. France 115 (1987), 361-390.

[We] A. Weinstein, Lectures on symplectic manifolds, CBMS Regional Conf. Ser. in Math., no. 29, Amer. Math. Soc., Providence, R.I., 1979.

[Y] J. A. Yorke, Periods of periodic solutions and the Lipschitz constant, Proc. Amer. Math. Soc. 22 (1963), 509-512.

[Y-S] V. A. Yakoubovich and V. M. Starzhinskii, Linear differential equations with periodic coefficients, Wiley, New York.

Ceremade, U.A. 749, P Ce du M Al de Lattre de Tassigny F-75775, Paris Cedex 16 , FRANCE

Current address: MSRI, 1000 Centennial Drive, Berkeley, California 94720 